\title{
Crecimiento económico y desigualdad de género: análisis de panel para cinco países de América Latina
}

\author{
Alison Vásconez Rodríguez
}

\section{Resumen}

En el presente estudio se analiza la relación entre la feminización del mercado laboral - entendida como el incremento de la relación de participación laboral y las horas ofertadas de las mujeres en el mercado - y el crecimiento económico en cinco países de América Latina. Por una parte, este aporte se ve reflejado en el potencial de demanda que la participación puede movilizar hacia el crecimiento económico. Por la otra, las condiciones de entrada y permanencia de las mujeres en el mercado laboral determinarán otro nivel de aporte desde la oferta (costos). Se calculan funciones de oferta de trabajo y se incluyen las horas ofertadas, las tasas de feminización y los salarios estimados en funciones dinámicas de crecimiento económico. Las conclusiones apuntan a que la feminización laboral tiene una relación positiva con el crecimiento, no así el incremento en las horas ofertadas.

Palabras clave

Crecimiento económico, género, incorporación de la perspectiva de género, mujeres, empleo de la mujer, igualdad de género, modelos econométricos, América Latina

Clasificación JEL

B54, J22, J71, O40

Autora

Alison Vásconez Rodríguez es Profesora Asociada de la Facultad Latinoamericana de Ciencias Sociales (FLACSO), Ecuador. avasconez@flacso.edu.ec 


\section{Introducción}

La economía feminista propone dos elementos para el debate sobre la vinculación micro-macro de la desigualdad de género y el crecimiento económico. Primero, señala que las políticas y los fenómenos macroeconómicos se vinculan con las personas y los hogares, o se filtran hacia ellos, a través de un sistema de instituciones - entre las que se encuentran el mismo Estado, el mercado y la comunidad-, en donde opera un orden que no necesariamente responde a la automaticidad ni a la organización completas. Se trata de un orden de género, basado en un sistema de relaciones de poder que ha permitido que un conjunto normativo/político (visible, masculino) domine o ejerza influencia sobre el otro (invisible, femenino) (Carrasco, 2001 y 2003; Elson, 1995a y 1999; Blau, Ferber y Winkler, 2002). El arrastre del orden de género hacia el orden del mercado genera señales sesgadas en el mercado, donde se asume que las mujeres son trabajadoras "costosas" y por lo tanto hay resistencia a contratarlas y mantenerlas en el trabajo ${ }^{1}$. La decisión de ofertar trabajo en el mercado es, entonces, un proceso de negociación y conflicto, en donde tienen peso factores materiales e inmateriales que permiten o impiden que esta decisión tenga - en el caso de las mujeres - un resultado favorable, tanto en términos de participación en el mercado como de las condiciones de entrada y permanencia en el trabajo².

El segundo elemento tiene que ver con las relaciones entre la esfera del mercado (llamada económica) y la del ámbito doméstico (llamada no económica). En este debate se plantea que el proceso de acumulación utiliza las "energías humanas" como mercancías y el trabajo doméstico reproduce estas energías como parte integrante de las personas. Desde el punto de vista externo del hogar, estas energías se dirigen de la reproducción de las personas a la producción de mercancías. Desde el punto de vista interno del hogar, la producción de mercancías es el medio y la reproducción el fin (Picchio, 2003). En este sentido, la producción y reproducción forman parte del mismo proceso continuo; de hecho, el eje de la economía se encuentra, de acuerdo con estos postulados, desviado de su centro, que es la reproducción de la vida en la que el trabajo del ámbito doméstico no mercantil es el fundamento (Pérez, 2005; Carrasco, 2001).

En consecuencia, para entender las características del mercado laboral es necesario considerar las relaciones entre los dos ámbitos de trabajo y, ante todo, la lógica del trabajo doméstico, en especial en el caso de las mujeres, para quienes las tareas domésticas muchas veces definen la totalidad de sus acciones económicas y laborales. Las modalidades de ocupación de las mujeres - que pueden ser fruto tanto de la política laboral como de relaciones laborales - reflejan la tensión entre participación y organización del proceso de producción (Elson, 1995b), lo que ha sido "resuelto" a través de flexibilidad, segregación y diferencias salariales por igual trabajo ${ }^{3}$. Esto permite intuir, además, que las tensiones entre la economía mercantil y la llamada "economía del cuidado no remunerada" aumentan durante los períodos de crisis, muchos de los cuales también se caracterizan por la creciente necesidad de trabajadoras y trabajadores baratos que entran al mercado, en condiciones tales (flexibles) que

1 En varios estudios se muestra que este supuesto es más bien un mito, basado en sesgos de género en el mercado. Véase Berger y Szretter (2002).

2 Respecto de la temática de negociación y conflicto intrahogar, véanse Agarwal (1997) y Sen (2000).

3 El trabajo a tiempo parcial es mayoritariamente femenino (pese a que en las últimas décadas se han registrado incrementos sustanciales en la intensidad del trabajo, tanto en hombres como en mujeres) y flexible, aunque no de disponibilidad inmediata (lo que sí ocurre con los hombres). Además, es más "elástico". 
permiten responder a las necesidades de recuperación de las economías (Todaro y Yáñez, 2004) y en detrimento de la calidad de los procesos de reproducción y de la "calidad" de la fuerza de trabajo4.

Esto puede implicar que la llamada "feminización" de la fuerza de trabajo - es decir, la creciente participación de las mujeres en la oferta laboral-, aparte de tener consecuencias positivas en el bienestar y la autonomía económica de las mujeres, en la demanda efectiva y en el crecimiento, tenga incidencia en los ciclos económicos debido a las relaciones de género ${ }^{5}$. Esta incidencia se podría producir a través de, al menos, dos mecanismos que han sido empíricamente verificados: i) el trabajo doméstico no remunerado, que soporta el costo de la reproducción social, y ii) el trabajo de las mujeres, que produce, en promedio, lo mismo que el de los hombres por menos pago ${ }^{6}$.

Estos dos mecanismos pueden producir efectos diferenciados durante los ciclos económicos a través de dos canales (Darity, 1995; Erturk y Darity, 2000):

- Canal de la reproducción. Dado que el trabajo doméstico no remunerado genera un "ahorro" a la economía, la feminización puede tener un efecto negativo si este ahorro se reduce (al remunerar el trabajo doméstico por medio de servicios privados o estatales financiados por recursos fiscales, ya que parte del valor transferido a la economía en forma gratuita a partir del trabajo doméstico y de cuidados ya no pasaría al circuito económico, sino que incrementaría la masa salarial).

No obstante, el efecto puede ser positivo si en un período de crisis las familias "sustituyen" gastos por trabajo no remunerado. Es decir, si el ahorro requerido para la recuperación recae en el trabajo doméstico, frente a restricciones fiscales o prestaciones de la política social, o frente a situaciones de reducción de ingresos laborales por desempleo (Cerrutti, 2000).

- Canal del mercado laboral. La creciente participación femenina podrá tener un efecto positivo debido a la reducción de costos salariales (la mayor oferta de trabajo a menores salarios tiende a bajar los salarios medios), dada la desigualdad salarial por razón de género ${ }^{7}$.

Por otra parte, el efecto de la brecha salarial y la presión hacia abajo del salario medio puede ser negativo a nivel de la demanda efectiva. Ambos casos pueden darse a la vez y el efecto neto dependerá de la estructura de la economía (Blecker y Seguino, 2002).

Para Darity (1995), los efectos del canal reproductivo pueden ser mayores que los del mercado laboral para los países más pobres y con menos servicios públicos de cuidado. Lo opuesto ocurriría en países más ricos. En esta línea, Erturk y Cagatay (1995) sistematizan diversos estudios empíricos sobre feminización laboral y, siguiendo a Rubery (1988), identifican tres modalidades de inserción laboral de las mujeres:

4 Este deterioro podría atenuarse mediante el aumento de la productividad en la economía doméstica no remunerada, así como el traspaso (mercantilización o socialización) de algunas de sus actividades. Lo primero es una tendencia, en mayor o menor ritmo, de las economías (y los distintos sectores que las integran) de acuerdo con su nivel de ingreso. Lo segundo depende más de los momentos del ciclo económico y del régimen de bienestar vigente en los países. No obstante, los servicios de cuidado remunerados han sido actividades muy feminizadas y generalmente mal remuneradas, reflejo de la poca valoración social de las actividades reproductivas. El acceso a estos servicios, por otra parte, también depende del nivel de ingreso familiar.

5 El debate sobre crecimiento desde el enfoque de demanda efectiva puede revisarse en Dutt (1990), Lo Vuolo (2009) y Bhaduri (2007).

6 La evidencia sobre brechas salariales por razón de género es extensa en América Latina. Más adelante se analizará un grupo relevante de estos estudios.

7 A nivel global, estos procesos de feminización laboral y sus condiciones han configurado estructuras desiguales en los mercados internacionales de trabajo y procesos de informalización debidos a las necesidades del capital (véase Benería, 2005 y 2008). 
- Una primera modalidad, de tipo procíclico (buffer), en la que la mano de obra de las mujeres se comporta como un ejército de reserva flexible que se incrementa en crecimiento y disminuye en recesión.

- Una segunda modalidad, segmentada, en la que existen sectores feminizados y el impacto de las recesiones o los auges de la economía en la composición de la fuerza de trabajo (entrada o salida de mujeres), depende de la composición de los sectores y las ocupaciones que acompañan estos ciclos. La estructura feminizada "protege" a las mujeres de ser las primeras en salir del mercado en situaciones de recesión. En este sentido, hay un comportamiento contracíclico.

- Una tercera modalidad que indica que la mano de obra de las mujeres sustituye la mano de obra masculina en contextos de crisis, cuando los principales perceptores pueden estar en situación de desempleo o subempleo. Igualmente, el mercado puede inclinarse por cooptar personas con menores expectativas de salario, pero que puedan realizar un trabajo similar, con el fin de ahorrar costos en una situación recesiva. La existencia de desigualdades de género podría operar en función de la recuperación económica, si su efecto en el costo es mayor que las consecuencias de la desigualdad sobre la demanda efectiva.

Este artículo está organizado en cinco secciones, incluida esta Introducción. En la segunda sección se presenta una revisión de la literatura referida al vínculo entre crecimiento y desigualdad de género. En la tercera se especifica la metodología empleada y en la cuarta los resultados obtenidos a partir de dicha metodología. En la quinta sección se brinda un conjunto de reflexiones a modo de conclusión.

\section{Literatura sobre crecimiento y desigualdad de género}

Hay dos grandes grupos de estudios que analizan la relación entre desigualdad de género y crecimiento. El primero tiene que ver con la desigualdad en capital humano, particularmente en educación, en tanto que el segundo está relacionado con el empleo y los ingresos laborales.

En relación con la desigualdad educativa, existe una extensa cantidad de investigaciones que muestran que la desigualdad de género desacelera el crecimiento a largo plazo y encuentran una relación positiva entre la educación de las mujeres y el crecimiento (Dollar y Gatti, 1999; Klasen, 2002). La interpretación de estos resultados apunta a que, al tener hombres y mujeres la misma dotación y distribución de talentos y capacidades, la desigualdad excluye a mujeres potencialmente bien calificadas y puede incluir a hombres menos calificados. Si se excluye a las mujeres a través de una menor educación y se da más educación a los hombres, en un ambiente de retornos decrecientes, educar a las niñas tendrá mayor retorno marginal. Este conjunto de estudios tienen como base uno de los principios que sustenta la teoría del capital humano, que propone una línea directa entre la inversión educativa y el crecimiento económico, intuyendo, aunque sin una verificación rigurosa, que la educación (formal) incrementa la productividad. Entre otras cosas, esta teoría ha sido cuestionada partiendo de que la productividad no es observable y los ingresos, así como la demanda de empleo, contienen sesgos de género que no tienen que ver con la dotación educativa. Por ende, si bien los resultados estarían mostrando que la desigualdad educativa puede ser perjudicial para el crecimiento (ya que se estaría perdiendo un potencial de mano de obra calificada), no necesariamente revelan que si las mujeres invierten en educación (o alcanzan un mayor nivel educativo) tendrán mayores ingresos y accederán a trabajos de mayor productividad. 
Otra vía de impacto de la desigualdad educativa que se discute desde la evidencia empírica son las externalidades que la educación de las mujeres genera a través de menores tasas de fecundidad, mortalidad infantil y educación de la generación futura (Cavalcanti y Tavares, 2007). La alta fecundidad también puede reducir la tasa de ahorro de largo plazo y la inversión en educación y salud (Klasen, 2002; King y Porter, 2010). Estas conclusiones son bastante intuitivas, dado que en la mayoría de los casos no se incluye la última variable (educación futura), y en la primera variable puede haber problemas de endogeneidad que no son lo suficientemente tratados ${ }^{8}$. Por otra parte, en la mayoría de estos estudios se utilizan regresiones de corte transversal e implícitamente se asume que el papel de la desigualdad de género es similar en todos los países, ignorando las características estructurales de cada uno. La inclusión de estudios de panel mejora mucho estas investigaciones, por lo que algunos autores (Klasen y Lamanna, 2009; Klasen, 2006) incluyen datos de panel en las actualizaciones de sus estudios. Las conclusiones en esencia no cambian, pero los resultados son más robustos.

En esta misma línea, otra vía de impacto es la de la competitividad. En economías orientadas a exportaciones intensivas en mano de obra femenina, la educación de esta mano de obra es clave para elevar la competitividad. Así, se encuentra una alta correlación entre la educación de las mujeres y las exportaciones (y, por ende, el crecimiento económico) (Seguino, 2000). Esta línea argumentativa tiene poca evidencia empírica, en esencia por un problema de información sobre la competitividad y la productividad interna en los países. En economías más orientadas hacia la agricultura, el efecto de género en el crecimiento está más vinculado a las desigualdades en la propiedad de la tierra y el crédito que a las desigualdades educativas (Blackden y Bhanu, 1999; Doss y Morris, 2001). En estos casos, los indicadores más demostrativos sobre inequidad de género son los de brechas en educación primaria, acceso a crédito y derechos de propiedad.

En relación con las desigualdades salariales y el empleo, los estudios son menos abundantes y sus resultados responden a diferentes marcos teóricos y, por ende, han dado paso a un importante debate. En un grupo de investigaciones se pone énfasis en los determinantes de largo plazo del crecimiento de la productividad y el enfoque de la oferta. En esta línea, en algunas investigaciones se modelizan la maximización de la ganancia de los productores mediante la selección de un conjunto adecuado de capitales humanos, cuya disponibilidad se ve reducida por la no entrada al mercado de personas potencialmente productivas (Esteve-Volart, 2004). En estos estudios se toma la no inclusión de las mujeres en el empleo como una distorsión que reduce el conjunto de talentos, en el caso de empleados, y la productividad, en el caso de desigualdad en el acceso a tecnología y otros recursos productivos (Blackden y otros, 2007).

Klasen (2002) y Klasen y Lamanna (2009) desarrollan modelos de crecimiento económico en donde se incluyen, en forma consecutiva, variables de brechas de participación y educación para medir los efectos indirectos y ecuaciones que relacionan estas brechas con algunos determinantes del crecimiento, como la inversión. No obstante, no se puede distinguir si el efecto de la equidad en la participación en el crecimiento se debe a un estímulo de las ganancias resultante de los bajos salarios relativos de las mujeres (al ingresar al mercado de trabajo pueden reducir el salario medio y, por ende, el denominado "salario de eficiencia"). La posibilidad real de que los incrementos en la productividad del trabajo femenino se traduzcan en aumentos salariarles insesgados dependerá de situaciones estructurales de la economía y sus instituciones, que pueden reducir o incrementar su capacidad de negociación salarial.

De este modo, una plausible relación entre la desigualdad de género en el empleo y el crecimiento es la que proviene de las brechas salariales. Las brechas en el empleo en presencia de salarios más

\footnotetext{
8 Aguero y Marks (2008) y Cruces y Galiani (2007), entre otros, realizaron estudios sobre la relación entre fecundidad y oferta de trabajo de las mujeres.
} 
bajos de las mujeres pueden reducir el crecimiento porque se pierde la oportunidad de utilizar la mano de obra más barata como ventaja competitiva (Seguino, 2000). En este caso, la desigualdad salarial -y no la igualdad - es eficiente. Esto coincide con la evidencia de crecimiento de algunos países, entre ellos los latinoamericanos, que han experimentado períodos de crecimiento acompañados de desigualdad, reducción del salario real o desempleo (este es el caso, por ejemplo, de los países del área andina en los primeros años de la década de 2000). No obstante, no hay suficientes estudios empíricos que respalden esta argumentación.

Un segundo grupo de investigaciones enfatiza los efectos del "lado de la demanda" de la desigualdad en salarios y empleo, tanto a corto como a largo plazo (Erturk y Cagatay, 1995; Blecker y Seguino, 2002). Estas investigaciones ponen énfasis en las características estructurales de las economías, que influyen en la interacción entre las relaciones de género y los agregados macroeconómicos. En estos estudios se da cuenta de que en países semiindustrializados las diferenciales salariales pueden estimular dos importantes agregados económicos: la inversión y las exportaciones (Seguino, 2000 y 2010). En países en desarrollo, muy dependientes de las exportaciones para financiar sus importaciones de bienes de capital, el trabajo femenino ha sido una de las principales fuentes de ganancias de tipo de cambio y de relajación de la restricción externa (debido a ganancias en competitividad).

En algunos estudios se ha intentado incorporar dimensiones que den cuenta de diferencias de género en modelos de crecimiento, y en especial en los patrones de oferta de trabajo diferenciados que reflejen la dependencia de las actividades de cuidado, así como la tendencia de segregación de las mujeres hacia actividades con alta intensidad de mano de obra en sectores primario exportadores. En este contexto, Blecker y Seguino (2002) encuentran que los mayores salarios pueden estimular el consumo agregado (asumiendo una mayor propensión al consumo de las trabajadoras que de los capitalistas) y producir un estímulo en la demanda agregada. Estos salarios, no obstante, también pueden generar incrementos en los precios de las exportaciones, en cuyo caso la demanda exportable decrece. Además, los salarios elevados reducen las ganancias en el sector, lo que puede resultar en un descenso de la inversión. Este efecto de demanda negativo (en exportaciones e inversión) es potencialmente mayor que el estímulo en consumo, en especial si las exportaciones son elásticas al precio (como sucede con los productos básicos).

Por otra parte, además de la competencia por medio de los salarios, en economías guiadas por la exportación, otro posible vínculo con el crecimiento es que las mujeres están concentradas también en los sectores de producción de alimentos (agricultura doméstica), y, por ende, los salarios más bajos en estas industrias pueden traducirse en precios bajos de la canasta de alimentos. Esto, a su vez, reduce los salarios medios en la economía como un todo y genera mayor competitividad (Kucera, 2002) y posibles efectos exportables positivos. Para analizar esta y otras vías sería necesario contar con una muestra amplia de países e información detallada sobre su estructura de demanda interna.

Tanto la evidencia como las aproximaciones intuitivas de estos estudios muestran que los efectos del crecimiento en la equidad de género pueden ser contradictorios: la desigualdad de género puede estimular algunos agregados económicos, pero también puede tener efectos negativos en otras áreas, como el consumo, si pesan los efectos de la demanda (Stotsky, 2006; Berik, Rodgers y Seguino, 2008; Braunstein, 2008). Las iniciativas orientadas a incrementar los salarios de las mujeres en función de una mayor equidad pueden conducir al crecimiento en un régimen no guiado por la ganancia, pero también pueden generar una contracción económica debido a efectos negativos en la inversión y las exportaciones. Estas perturbaciones de corto plazo en la demanda no solo pueden cambiar el empleo masculino y el producto, sino que también pueden desacelerar la llegada del crecimiento de largo plazo. Por consiguiente, si la equidad de género produce efectos de oferta positivos en la calidad de la fuerza laboral en el largo plazo, en el corto plazo esta equidad de género 
puede provocar choques que pueden sacar a la economía de su ruta. La duración de este desvío debería analizarse (Berik, Rodgers y Seguino, 2009).

En el presente estudio se pretende verificar, para el caso de un grupo de países de América Latina, la relación entre el crecimiento económico y la feminización de la fuerza laboral (definida por la participación de las mujeres en el mercado laboral), de acuerdo con el planteamiento de Erturk y Cagatay (1995) descrito en la sección I. Los autores plantean que cuando hay bajos niveles de actividad (o cuando la economía está en recesión), la tasa de feminización crece, asumiendo un carácter contracíclico. A medida que esto ocurre, la feminización estimula la inversión (dados los salarios bajos y la limitada capacidad de negociación, lo que genera brechas salariales). La inversión se eleva y empuja el equilibrio bajo hacia uno más alto. A la vez, la intensidad en el trabajo doméstico impacta en el ahorro de los hogares, que dejan de gastar en servicios de cuidado. Si en la base del ciclo este último efecto tiene mayor influencia que el del trabajo mercantil, la feminización provocaría presiones sobre la utilización de la capacidad hacia abajo y profundizaría la recesión. Lo contrario ocurre si, cuando la economía está en contracción, la inversión tiene más incentivo frente al costo que el ahorro frente al trabajo doméstico.

En este estudio se recogen estos postulados. No obstante, sobre la base de la evidencia empírica en América Latina (Contreras, Armas y Vásconez, 2008; Vásconez, 2012; Esquivel, 2005), se asume que, si bien la participación laboral es contracíclica y también el trabajo doméstico es más intenso entre la población con menos ingresos y menos acceso a servicios privados de cuidado, lo que ocurre en recuperación es menos claro, al igual que el efecto que la feminización laboral tiene en el ciclo económico. Dado que la respuesta del trabajo doméstico ante recesiones económicas no puede verificarse empíricamente, se puede asumir, por falta de información histórica, que la feminización del mercado de trabajo, en términos de intensidad (horas de trabajo) en un cierto rango, sustituye el tiempo dedicado al trabajo de cuidados, y ello puede tener las consecuencias de desahorro descritas en el marco conceptual. Si bien esta investigación se enfocará en la relación entre la feminización del mercado laboral y el crecimiento económico, y no incluirá datos seriados sobre el uso del tiempo para el trabajo doméstico, se incorporará la variable de horas de trabajo como una variable sustitutiva de la relación trabajo de mercado-trabajo doméstico, con la finalidad de intuir un comportamiento de la oferta de trabajo globalmente considerada. Los resultados darán información sobre el denominado "canal del mercado" en el marco conceptual utilizado.

\section{Metodología}

Se especifica que la oferta laboral de los individuos depende del salario ofrecido y el salario de reserva, que incluye el tiempo de cuidados y el tiempo disponible del otro perceptor. Es decir, la oferta laboral mercantil se inicia cuando el salario ofrecido es mayor que el salario de reserva. Suponiendo que se trata de un hogar nuclear, la oferta laboral de la persona $m$ está relacionada con el salario de su pareja $h$.

$$
\begin{gathered}
H_{m}=0 \quad \text { si } w_{m}<W R_{m} \\
H_{m}=H_{m}\left(w_{m}, W R\right) \quad \text { si } \quad w_{m}>W R_{m}
\end{gathered}
$$

Donde $W R=W R\left(p, T d_{m}, y / p, w_{h}\left(T-T d_{h}\right)\right)$ es el salario de reserva, que incluye tanto las condiciones laborales de quien oferta trabajo como del "otro perceptor", para dar cuenta de una decisión conjunta. $H_{m}$ es la oferta laboral tal como se describe en (3) y $\varepsilon_{m}$ es el residuo. 
Como solamente se pueden ver los salarios de las personas que participan, existe un sesgo de selección muestral. Entonces, para definir la oferta laboral corrigiendo este sesgo se sigue el método de tres etapas de Heckman ${ }^{9}$. Primero, se calcula el sesgo de selección mediante un modelo de participación reducido (es decir, sin el salario). Considerando una función general de oferta laboral sin salario, de acuerdo con Heckman (1976 y 1993), este sesgo está dado por la relación entre la densidad de probabilidad y la probabilidad acumulada de la función de oferta laboral en su forma reducida. A esta razón también se la denomina relación inversa de Mills (o lambda, $\lambda$ ), cuyos valores medios representan una variable sustitutiva de la existencia de barreras de entrada al mercado laboral. Con la inclusión del sesgo, se calcula el salario incluyendo la relación inversa de Mills como un regresor:

$$
\hat{w}_{i}=X_{i} \beta+\theta \lambda_{i}+v
$$

El tercer paso es estimar la oferta laboral mediante una regresión de mínimos cuadrados, ya que se trata de la oferta en horas, incluido el salario estimado. La especificación formal es la siguiente, en modelos separados para hombres (h) y mujeres $(m)$ :

$$
\begin{gathered}
H_{m i}=\beta_{m}+X_{m i}{ }^{\prime} \beta_{1, m}+Z_{m i}{ }^{\prime} \beta_{2, m}+D_{m i}{ }^{\prime} \beta_{3, m}+Y_{m i}{ }^{\prime} \beta_{4, m}+w_{i m}{ }^{\prime} \eta_{m}+\varepsilon_{m i} \\
H_{h j}=\beta_{h}+X_{h j}{ }^{\prime} \beta_{1, h}+Z_{h j}{ }^{\prime} \beta_{2, h}+D_{h j}{ }^{\prime} \beta_{3, h}+Y_{h j}{ }^{\prime} \beta_{4, h}+w_{h j}{ }^{\prime} \eta_{h}+\varepsilon_{h j}
\end{gathered}
$$

Donde $\mathrm{X}, \mathrm{Z}, \mathrm{D}$ e $\mathrm{Y}$ son vectores de capital humano, condiciones laborales, condiciones demográficas e ingresos, y w es un vector de salarios.

En el caso del modelo probabilístico, se deben calcular los efectos marginales dados por $\partial \mathrm{H} / \partial \mathrm{lnw}$, que muestra el cambio en la probabilidad de participación debida a un cambio porcentual en el salario (que no es la elasticidad directa). La sumatoria de las probabilidades estimadas como proporción del total de población en edad de trabajar indica la tasa neta de participación estimada a nivel agregado. La elasticidad es la razón del cambio porcentual en el salario sobre el cambio en esta tasa.

Una vez obtenidas las tasas de participación, se calcula el indicador de feminización, que es la razón de participación de mujeres frente a hombres. Con estas variables se construyen tres modelos de crecimiento que tienen como variable dependiente la tasa de crecimiento del producto interno bruto (PIB) y en los que se incluyen alternativamente las variables de feminización laboral y horas de trabajo. También se incluyen variables que dan cuenta del crecimiento de la intensidad del trabajo, el salario y las brechas educativas, en línea con otros estudios presentados en la revisión bibliográfica. Reconociendo la endogeneidad de la relación entre desigualdad y crecimiento explicada en el marco teórico, se corrigen estos problemas utilizando rezagos de las variables explicativas y de la variable dependiente, así como instrumentos dados por diferencias entre los valores de períodos anteriores, de conformidad con el método generalizado de momentos.

La naturaleza del análisis de la relación entre crecimiento y desigualdad de género, así como la relación de causalidad planteada en el marco conceptual presentado, requiere de un análisis que vincule las brechas de género en el mercado laboral y sus consecuencias en el crecimiento de la economía (en este caso, representado por el crecimiento del producto). Por ello, resulta más adecuado incluir variables que tengan en cuenta sus valores anteriores, en especial en aquellos casos de interés para el presente análisis. Si bien no se va a realizar un análisis de convergencia en crecimiento, se parte de un modelo dinámico autorregresivo para explicar sus consecuencias en el modelo econométrico de

\footnotetext{
9 Véase una discusión sobre los diferentes tipos de modelos de oferta laboral en Berndt (1990), Killingsworth (1983) y Blundell, MaCurdy y Meghir (2007).
} 
panel, y de allí se incorporan otras variables rezagadas, con el fin de explicar el modelo que se va a utilizar en la investigación.

En esta investigación se utiliza un modelo de panel dinámico no autorregresivo, en el cual la variable dependiente es la tasa de crecimiento trimestral del PIB para cada país. Como explicativas se proponen cuatro conjuntos de variables:

i) variables macro relacionadas con el crecimiento ${ }^{10}$;

ii) variables del mercado laboral;

iii) variables de brechas de género;

iv) variables de país.

Se proponen tres especificaciones. En la primera, el conjunto de variables macro incluye: el crecimiento del PIB rezagado, la inversión como proporción del PIB y el crecimiento del capital humano medido por la escolaridad media de la población económicamente activa (PEA). En el conjunto de variables laborales se incluye el crecimiento de la oferta laboral (horas ofertadas) y del salario medio. También se incluyen variables ficticias de país combinadas con la tasa de feminización, para poner énfasis en los efectos individuales. En la segunda especificación se incorporan como controles en el conjunto macro el nivel de apertura económica e industrialización rezagados, en el laboral el crecimiento del salario y en el capital humano la brecha de género de escolaridad. En una última etapa se completa el modelo con la inclusión de dos variables de contexto específico de los países, una que tiene que ver con períodos de cambio de gobierno (en todos los casos, salvo el Brasil, ocurrieron cambios en el período de estudio 2003-2010, por lo cual en este país no se incluye esta variable) y períodos de crisis, que corresponden a 2003 en la Argentina y el Uruguay, y al segundo y tercer trimestre de 2009 (la crisis financiera reciente) para todos los países.

Se realiza primero una prueba de Hausman para verificar efectos fijos. Los resultados arrojan un valor del estadístico bastante inferior al de las tablas chi-cuadrado, con lo cual se rechaza la hipótesis nula de que no existe diferencia significativa en los dos tipos de modelos (véase el anexo A1). Esto permite afirmar que hay una correlación entre las variables explicativas y el efecto propio, es decir que se trata de un caso en el que las diferencias entre los países son clave para entender el comportamiento de la variable dependiente.

Posteriormente, de acuerdo con la metodología planteada, se corre el modelo no separado (pool) a través de mínimos cuadrados ordinarios (MCO) para verificar la cota superior del estimador. A continuación, se obtienen los estimadores within, con variables en diferencias y sin la inclusión de instrumentos de momentos para las variables independientes.

Por último, se corre el modelo a través del método generalizado de momentos (MGM SYS). La especificación formal es:

$$
Y_{i t}=Z_{i t}{ }^{\prime} \beta_{1}+G_{i t}{ }^{\prime} \beta_{2}+L_{i t}{ }^{\prime} \beta_{3}+D_{i}{ }^{\prime} \beta_{4}+P_{i t}{ }^{\prime} \beta_{5}+\varepsilon_{i t}
$$

Donde $Y_{i t}$ es la tasa de crecimiento económico, $Z_{i t}$ es el vector de variables macro, $G_{i t}$ es el vector de variables de "género" donde se incluye a las de interés, $L_{i t}$ es el vector de condiciones del mercado laboral, $D_{i}$ está formado por las variables ficticias de país combinadas por sus tasas de feminización y $P_{i t}$ está conformado por las variables ficticias correspondientes a factores exógenos (crisis y cambio de gobierno). Se realizan las siguientes consideraciones para los instrumentos:

\footnotetext{
10 La selección de estas variables se ha basado en varios estudios sobre estimaciones de crecimiento (Barro, 2000; Ros, 2004) y algunos de los trabajos que se describen en la revisión bibliográfica.
} 
- Variables endógenas: feminización laboral y su tasa de crecimiento (considerando que existe una relación bidireccional entre el crecimiento y la desigualdad), crecimiento de la feminización laboral, crecimiento del salario en el período anterior y brechas salariales. Estas se instrumentan a través de diferencias y nivel. La verificación de la endogeneidad se realiza mediante una regresión, en forma reducida, de estas variables frente al crecimiento económico (véase el anexo A2). En macroeconomía, esta especificación se utiliza con la finalidad de destacar una relación de causa-efecto, dado que se han rezagado y diferenciado las variables explicativas con la finalidad de superar la endogeneidad ocasionada - en este caso - por la bidireccionalidad de la relación (que se ha encontrado en los estudios descritos anteriormente). La interpretación, una vez sometida a prueba la no endogeneidad, puede hacerse señalando que cierto cambio en la variable de feminización laboral tiene determinado efecto en la tasa de crecimiento económico.

- Variables predeterminadas: tasa de inversión, escolaridad de la PEA y brecha de género en escolaridad. Estas se instrumentan a través de diferencias. Para seleccionar estas variables como débilmente exógenas se realiza la misma prueba que en el caso anterior (véase el anexo A2).

- Variables exógenas con rezago: variables macro: tasa de inversión, apertura económica y grado de industrialización. Se incluyen estos rezagos como sus propios instrumentos.

- Variables exógenas contemporáneas: variables ficticias.

La variable de interés es el indicador de feminización laboral. También se realizan las pruebas de Arellano y Bond para verificar la no autocorrelación de segundo orden y la prueba de Sargan de sobreidentificación.

\section{Fuentes de datos}

Para los datos macro, la información de crecimiento se obtiene de dos fuentes: i) metabase Penn World Table (PWT 7.0), y ii) CEPALSTAT. Para los modelos se utilizan datos trimestrales entre 2003 y 2010 que provienen del compendio estadístico "América Latina el Caribe: series históricas de estadísticas económicas 1950-2008” (CEPAL, 2009b), CEPALSTAT y las instituciones de estadística de los diferentes países (para valores tanto constantes como corrientes, con el fin de presentar toda la información en dólares constantes de 2000). La heterogeneidad entre los países analizados agrega más variabilidad, de modo que la muestra de datos resulta interesante para el análisis.

Para la derivación de la oferta laboral y el salario estimado se ha realizado una homologación de los microdatos de las encuestas de hogares de cinco países: Argentina, Brasil, Ecuador, Perú y Uruguay. Se han seleccionado estos países como un grupo interesante que muestra la diversidad de América del Sur: el Ecuador y el Perú en la región andina, con características sociales similares, pero económicas distintas; la Argentina, el Brasil y el Uruguay de la zona del Mercado Común del Sur (MERCOSUR), con la Argentina y el Uruguay con niveles económicos similares (ingresos relativamente altos), pero situaciones de pobreza y distributivas distintas, y el Brasil con un ingreso medio, alto crecimiento y situaciones de pobreza y distribución extremas. La selección del grupo de países también se debe a que se encuentran en puntos diversos en la relación feminización/PIB per cápita, de modo que se puede hacer inferencias interesantes, tanto grupales como individuales. Se obtuvo información de microdatos de las encuestas completas de empleo a nivel mensual desde 2003 hasta 2010 para las áreas urbanas. No obstante, se utilizan datos trimestrales desestacionalizados para tener una variabilidad adecuada frente a las variables macro. Sobre la base de estos resultados, para los modelos macro se ha conformado una base de panel de 32 períodos por país, correspondientes a los trimestres entre 2003 y 2010. 


\section{Resultados}

En el gráfico 1 se muestran las estimaciones de oferta laboral. La tendencia entre 2003 y 2009 da cuenta de un alza de dos puntos, en promedio, en la participación laboral femenina para el Brasil, el Ecuador y el Uruguay, y una reducción similar en la Argentina y el Perú.

\section{Gráfico 1}

Estimaciones de participación laboral

(En porcentajes)

Argentina

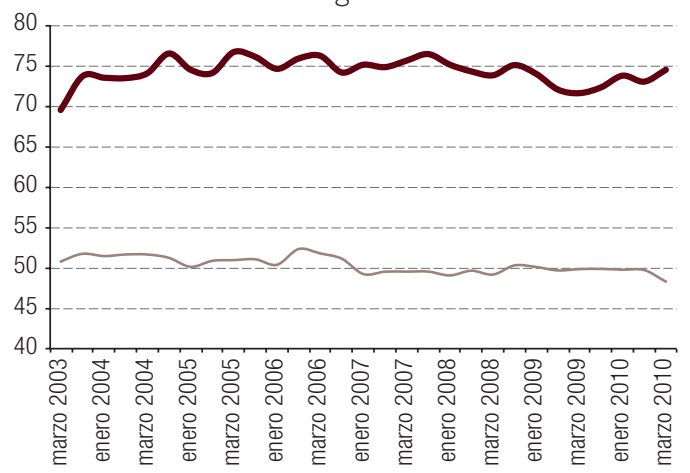

Perú

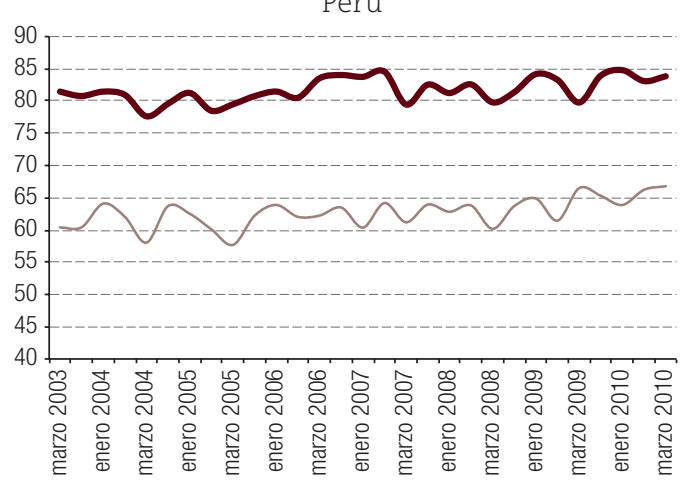

Ecuador

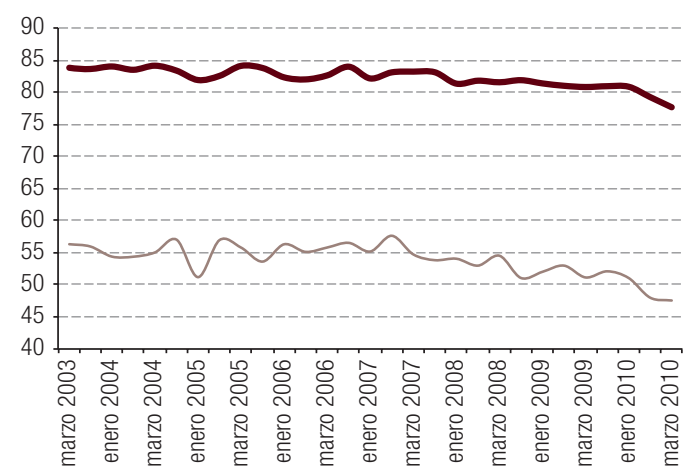

Uruguay

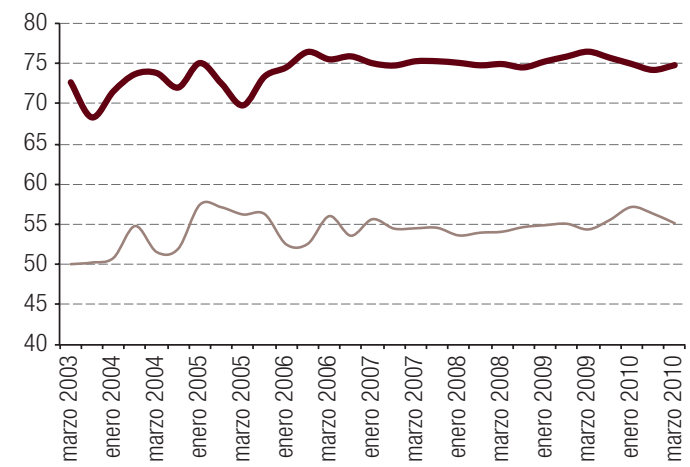

Brasil

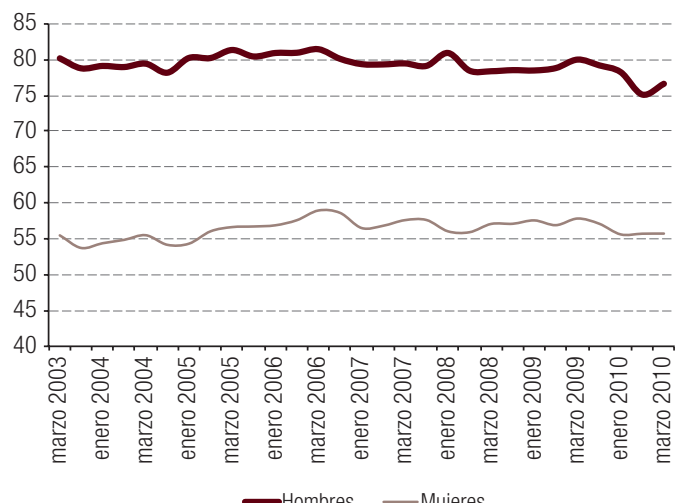

Fuente: Elaboración propia. 
De acuerdo con el marco conceptual planteado, el cálculo de la participación laboral en dos etapas permite analizar el sesgo por selección, a través de la relación inversa de Mills (lambda), cuyo significado se refiere a la tasa de riesgo (hazard rate) de no ser seleccionado. Los resultados de los modelos calculados permiten inferir que los valores de $\lambda$ son mayores para las mujeres que para los hombres, en una proporción de 1,5 a 1, en promedio, para la Argentina, 4 a 1 en el Ecuador y el Uruguay, y 2,5 en el Perú. Estas proporciones permanecen estables durante todo el período de estudio en el caso de la Argentina, se reducen en el Ecuador y el Perú, y se elevan en el Brasil y el Uruguay, dado un incremento en el valor para las mujeres entre 2007 y 2010, período en el que también aumenta el desempleo (véase el gráfico 2).

\section{Gráfico 2}

Valores estimados de $\lambda_{h}, \lambda_{m}$ (sesgo de selección)

Argentina

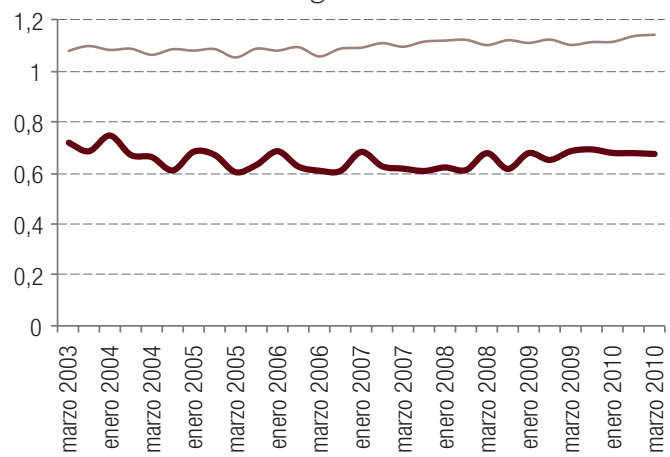

Perú

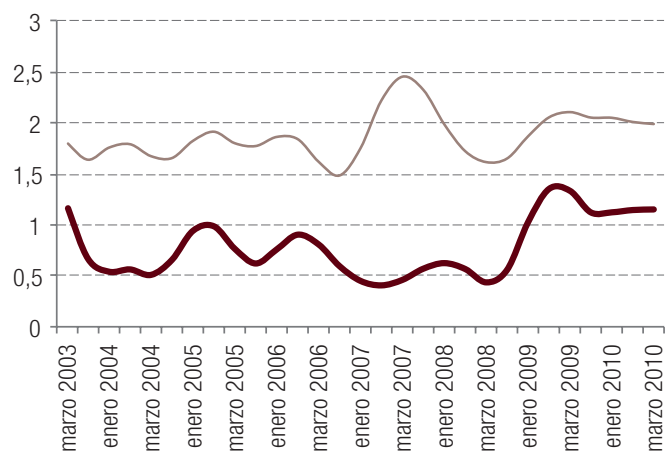

Ecuador

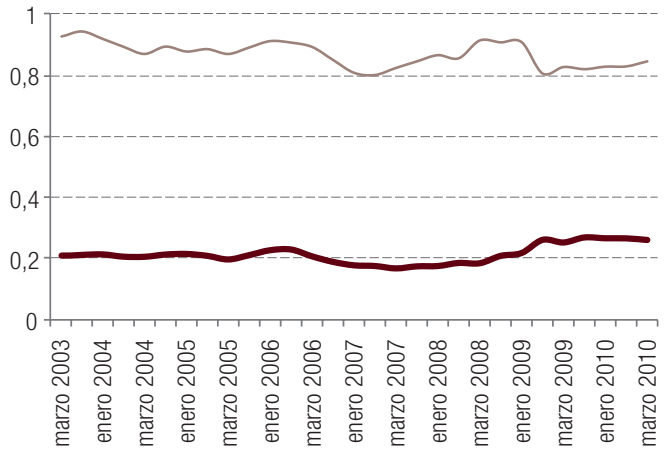

Uruguay

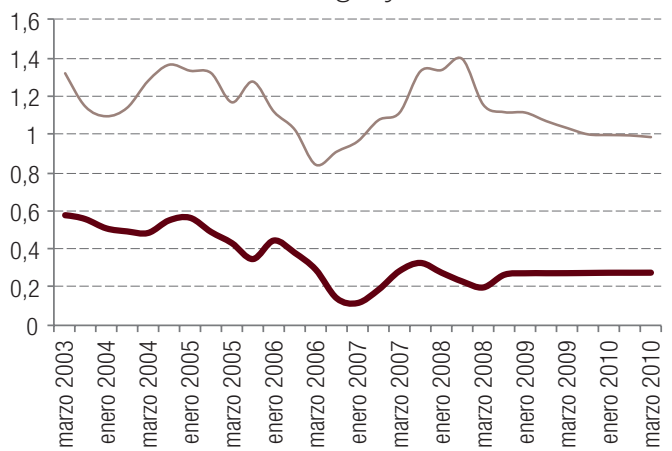

Brasil

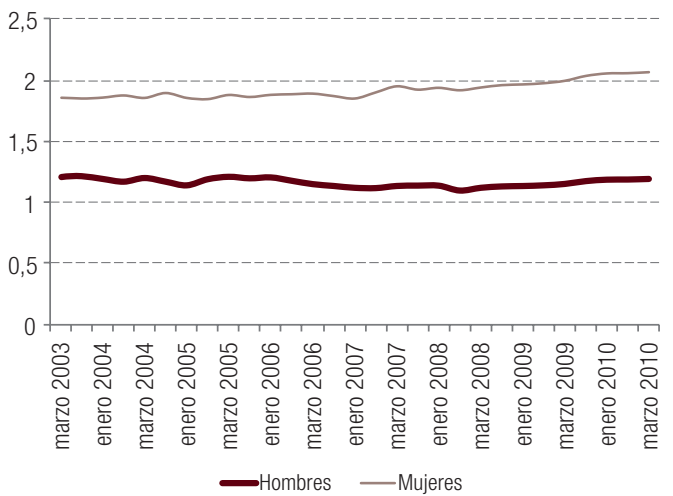

Fuente: Elaboración propia. 
Los mayores valores de lambda para las mujeres durante el período de estudio se presentan en el Brasil y el Perú, lo que no se refleja en la tasa de participación laboral de estos países (es decir, estos países no son los que tienen menor participación). Esto implica que, si bien la oferta laboral femenina es elevada (respecto de su población en edad de trabajar), gran parte de la no participación se explica por factores de selección, es decir que existe una oferta laboral potencial más alta que en los otros países. Esto podría estar en línea con la hipótesis de Erturk y Cagatay (1995), que plantea la existencia de una mayor intensidad de participación laboral en países tanto de alto como de bajo nivel de ingreso. Los menores valores de lambda se registran en el Brasil, pese a que la brecha frente a la selección masculina es la más alta. Esto puede relacionarse con la alta oferta de trabajo observada en los trabajadores, que se mantiene cerca del $80 \%$ durante la última década.

El movimiento del sesgo de selección tiende a ser inverso al desempleo en el caso de las mujeres. Lo opuesto ocurre para los hombres. Por ende, la mayor dificultad de entrada para las mujeres se da cuando el nivel de desempleo está bajando; es decir, cuando hay mayor probabilidad (ceteris paribus) de encontrar empleo o la economía está generando empleos. Asimismo, hay mayor entrada, menores barreras o mayor decisión de entrar (baja relativamente el salario de reserva) en períodos en que el desempleo está subiendo, lo que puede ser fruto de una desaceleración en la economía. Lo opuesto ocurre en el caso de los hombres, para quienes los valores de lambda se mueven paralelamente al desempleo, con lo cual se puede entender que su oferta laboral responde a una situación específica de oportunidad de empleo en el mercado. Esta situación, para ambos, se acentúa durante los últimos años de estudio, cuando se producen fuertes movimientos del desempleo de hombres y mujeres, en especial en la Argentina y el Ecuador (CEPAL, 2009a).

En el gráfico 3 se muestran las estimaciones de feminización del mercado laboral (cociente entre la tasa de participación estimada de mujeres y de hombres) y se puede observar una reducción de este indicador durante los cinco primeros años del período de análisis. En estos mismos años, la oferta femenina en el Perú, el Uruguay y el Brasil se eleva. A partir del tercer trimestre de 2009 hay un aumento de la feminización en todos los casos, salvo en el Ecuador. La tasa de crecimiento de la feminización tiende a estabilizarse solamente en la Argentina, moviéndose alrededor del 67\%. En los demás países se muestran grandes variaciones y al final del período la tasa más alta se registra en el Uruguay (75\%) y la más baja en el Ecuador (61\%), dada la alta participación masculina. Además, durante el período de análisis se mantiene una brecha media de 22 horas semanales ofertadas en el mercado, salvo en el caso del Ecuador, donde esta brecha es de 30 horas, en promedio, durante todo el período. En el Brasil se muestra una tendencia similar a la del Uruguay, pero más clara, hacia un incremento de la feminización, que acompaña al crecimiento del PIB hasta 2008. Si se observan las fluctuaciones de una y otra variable, no obstante, se puede constatar un comportamiento opuesto, en especial en los últimos años, lo que puede mostrar una cierta tendencia contracíclica, que requeriría un mayor período de análisis para su verificación. 


\section{Gráfico 3}

Feminización estimada del mercado laboral y crecimiento del PIB

(En porcentajes)

Argentina

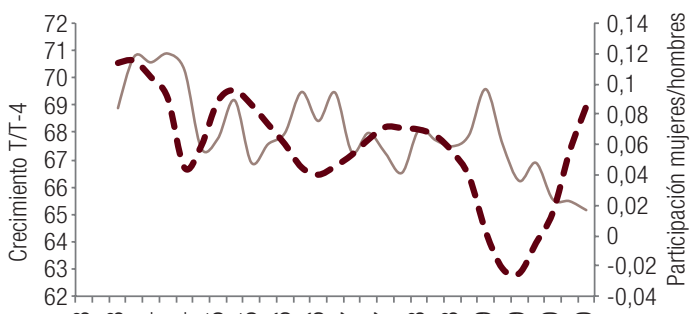

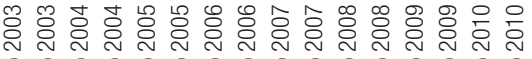

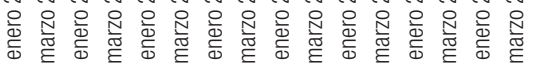

Perú

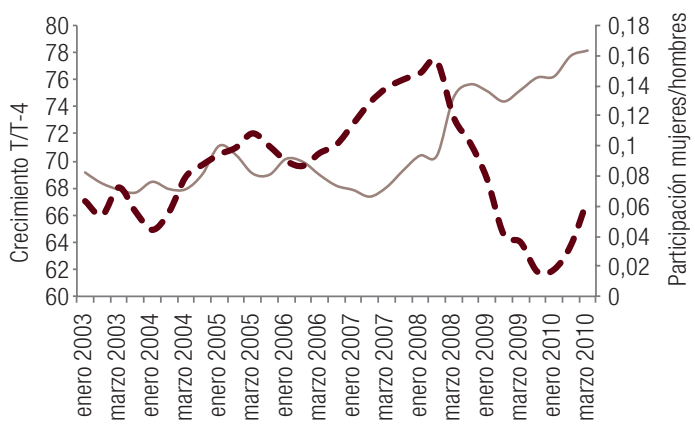

Ecuador

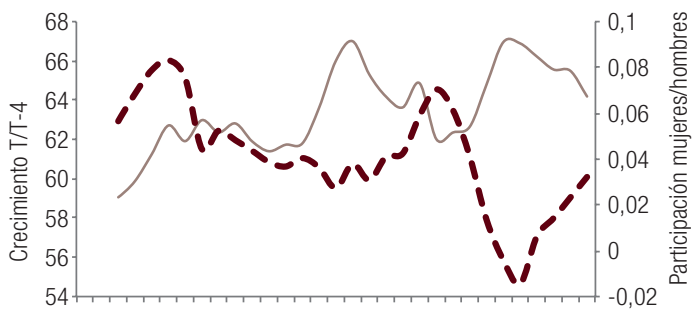

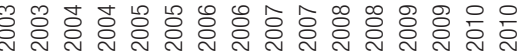

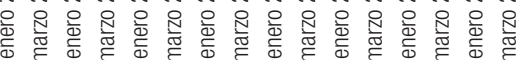

Uruguay

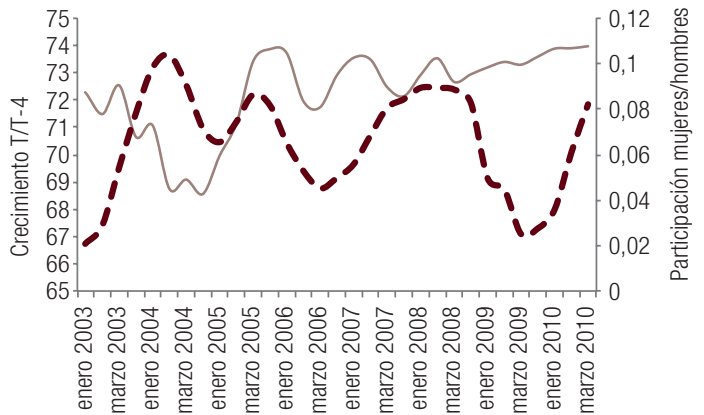

Brasil

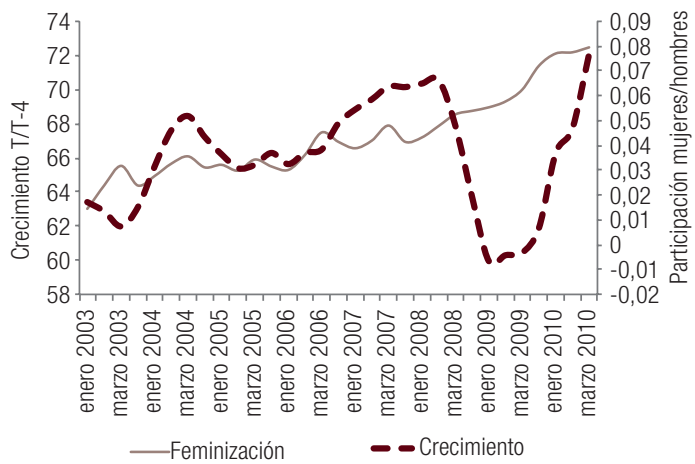

Fuente: Elaboración propia.

El incremento en la cantidad de personas en el mercado no implica un cambio en la oferta de horas de trabajo. La jornada laboral estimada para las mujeres corresponde, en promedio, a una dedicación parcial, lo que está acorde con la alta incidencia de subempleo entre la población femenina. De hecho, los incrementos de participación laboral que aparecen durante algunos períodos están acompañados de decrecimientos en la oferta de horas semanales. La mayor intensidad de trabajo en el caso de los hombres se registra en el Perú (alrededor de 50 horas semanales), con una reducción de 5 horas, en promedio, entre 2005 y 2007 (véase el gráfico 4). El Uruguay también presenta un promedio de dedicación mayor que la jornada regular de trabajo (alrededor de 45 horas durante todo el período). La menor intensidad se registra en la Argentina y el Brasil, en especial entre 
2003 y 2006 (27 horas en promedio). En este último caso se aprecia un incremento en la intensidad de trabajo de los hombres y un decrecimiento en el caso de las mujeres.

Gráfico 4

Oferta laboral estimada

(En horas por semana)
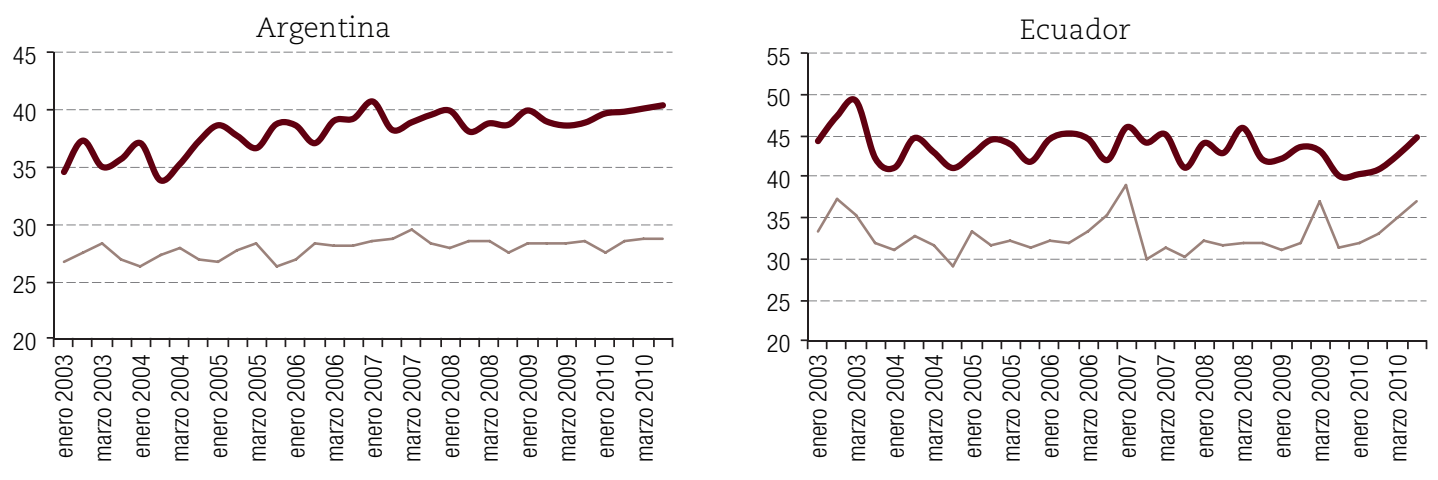

Perú
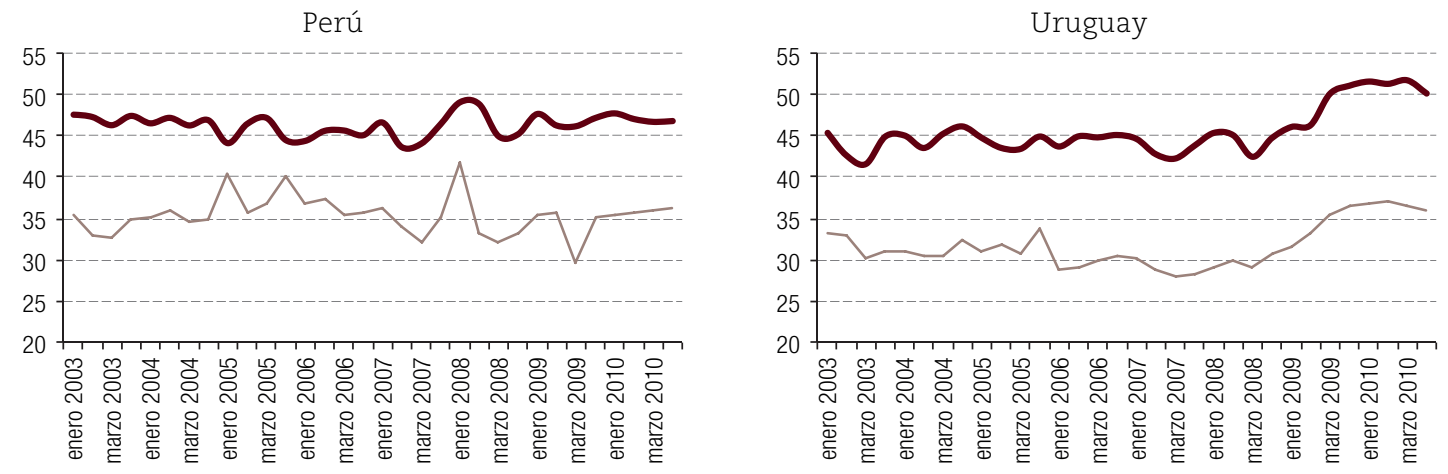

Brasil

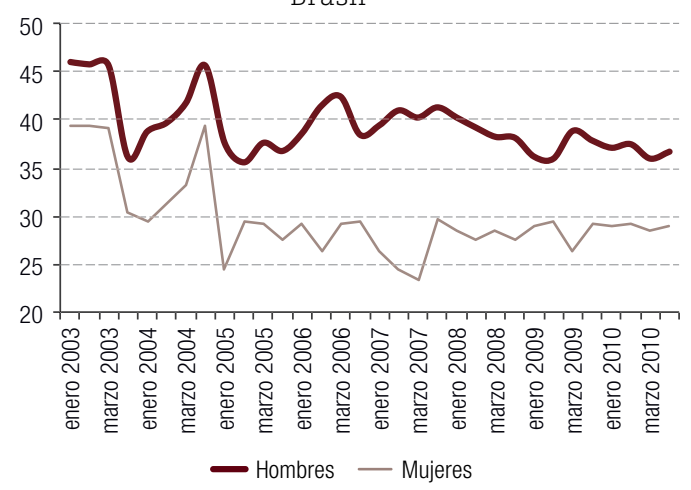

Fuente: Elaboración propia.

En cuanto a la relación entre la feminización laboral y el crecimiento económico, a continuación se presentan los resultados de los modelos descritos anteriormente, considerando de mayor validez la tercera especificación del método de momentos MGM SYS ${ }^{11}$ para corregir endogeneidad y

\footnotetext{
${ }^{11}$ Es decir, instrumentos obtenidos a través del método generalizado de momentos. Véase una explicación detallada de la justificación para el uso de este modelo en Martín (2008).
} 
exogeneidad débil ${ }^{12}$. Las estimaciones permiten confirmar la existencia de una relación significativa entre la feminización (medida en términos del cociente entre participación femenina y masculina) y la tasa de crecimiento económico. Los resultados se muestran para el conjunto de países y períodos analizados, ya que se trata de un modelo de panel. No obstante, también se incorporan variables específicas por país con la finalidad de detectar efectos específicos.

En el cuadro 1 se muestra que el incremento de 1 punto en la tasa de feminización se asocia con un incremento del 5,9\% en la tasa de crecimiento del PIB. Este efecto se mantiene y refuerza en el mediano plazo, ya que la variable rezagada de feminización en un período tiene un efecto del 7,7\% en la tasa de crecimiento. Este resultado se considera robusto dada la estabilidad tanto del coeficiente como del error estándar en las tres especificaciones. El resultado confirma, en parte, la hipótesis de que frente a una situación de recesión, o cuando la economía se encuentra en un nivel subóptimo de utilización de capacidad, la participación laboral femenina puede ayudar a la recuperación o al crecimiento, dada una brecha de participación laboral preexistente. Es decir, la reducción de estas brechas es positiva para el crecimiento de corto y mediano plazo.

\footnotetext{
12 Como resultado de la prueba de Haussman para efectos fijos o aleatorios, se obtuvieron valores del estadístico que permiten afirmar que se trata de un modelo de efectos fijos, de modo que se puede utilizar la metodología presentada anteriormente para este caso. En el cuadro 1 se pueden observar las corridas para el pool de mínimos cuadrados ordinarios y estimador intragrupos de efectos fijos, para las variables de interés. El resto de las variables se encuentran en el modelo detallado en el anexo A3. Las pruebas de Arellano y Bond para autocorrelación permiten no rechazar la hipótesis nula de ausencia de este fenómeno para el segundo orden en las ecuaciones en diferencias, con lo cual se justifica el uso de los instrumentos de momentos. La prueba de Sargan y Hansen, a su vez, verifica que no existe sobreidentificación. Además, se corrieron formas reducidas para evaluar el poder explicativo de los instrumentos adicionales (rezagos y diferencias) en las variables de interés. Los valores $\mathrm{F}$ obtenidos permiten confirmar la validez de los instrumentos.
} 


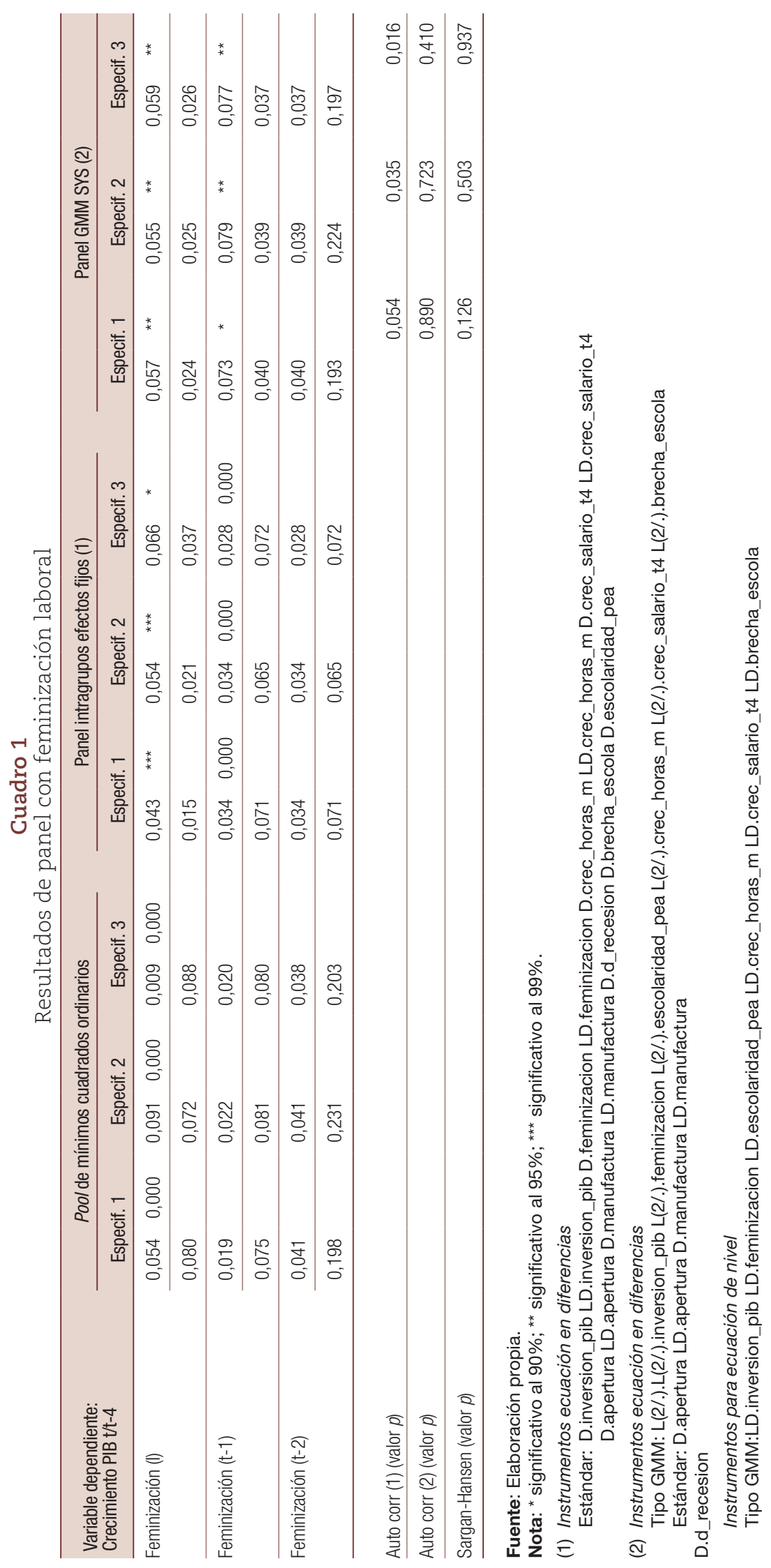


En el caso ecuatoriano, la evolución de la feminización durante el período de estudio se confirma con el resultado encontrado en el modelo conjunto. No obstante, las fluctuaciones de este indicador son opuestas a las fluctuaciones en la tasa de crecimiento. De ello se puede intuir que en este caso la participación femenina, que en conjunto impacta positivamente en la tasa de crecimiento, crece más rápidamente en presencia de desaceleraciones en la economía. Por otra parte, si bien la feminización tiene un efecto positivo en el crecimiento, cuando existe una desaceleración importante, como la del segundo y tercer trimestre de 2009 o los primeros meses de 2010 en algunos países, en presencia de mayor desempleo masculino, la reacción de la oferta femenina es positiva. De encontrarse este resultado en el largo plazo, se podría confirmar lo planteado por Erturk y Cagatay (1995) sobre la relación entre alta feminización y bajos ingresos per cápita.

En el Uruguay, la recuperación a partir del segundo trimestre de 2003 trajo un crecimiento sostenido que se ha visto menos afectado por la reciente crisis que en el resto de los países, y la participación laboral femenina continúa su crecimiento histórico a mayor velocidad que la masculina (Espino y Azar, 2005; Espino, Leites y Machado, 2009). Esto permite que las brechas de participación se cierren y la tasa de feminización se incremente, lo que se ajusta al resultado del modelo. Por otra parte, la brecha de desempleo se mantiene y amplía en 2007 y 2008, lo que quiere decir que la mayor participación no ha sido compensada con empleo.

En el caso del Brasil, el comportamiento del conjunto del mercado laboral muestra que en el reciente período de bonanza económica hay incrementos de la participación laboral de las mujeres, aunque los sesgos de selección se mantienen e incluso se incrementan al final del período. Además, aumentan las brechas de salario y se reduce el desempleo, mostrando mejores oportunidades de entrada y empleabilidad, pero no en empleos a tiempo completo. Es decir que los resultados sobre la feminización laboral son positivos tanto para las mujeres que ingresan al mercado como para el crecimiento económico, pero las condiciones de entrada y permanencia pueden no ser favorables para las mujeres, además de que el incremento de las brechas y la reducción del salario medio también podrían incidir en el crecimiento inmediato.

Los resultados grupales en la Argentina y el Perú se refuerzan con la inclusión de variables específicas de feminización por país, que tienen una relación positiva y significativa con la tasa de crecimiento. En el primer caso, un incremento en la tasa de feminización aumenta un 4,7\% la tasa de crecimiento del PIB. Este resultado está en línea con estudios recientes sobre el país a partir de la eliminación de la convertibilidad (Rodríguez, Esquivel y Espino, 2012), cuando el crecimiento sostenido del PIB fue acompañado por aumentos en la participación laboral de las mujeres, tanto por el crecimiento de los sectores donde hay mayoría de mano de obra femenina, como por la feminización de algunos sectores donde la participación femenina era menor en el pasado. También coincide con la convergencia que se observa en la participación masculina y femenina desde 2003 hasta 2007, los períodos de mayor crecimiento económico a partir de la recuperación. La tendencia a la convergencia de las tasas de ocupación en períodos de auge también coincide con la divergencia en las tasas de desempleo (Novick y Rojo, 2009).

En el caso del Perú, la feminización propia genera un incremento del 4,7\% en la tasa de crecimiento del PIB. La alta significancia de la variable de feminización propia puede deberse a lo ocurrido en los últimos años, cuando esta tasa se eleva sustancialmente durante el impulso de crecimiento ocurrido desde mediados de 2007. No obstante, esto ocurre cuando el salario de las mujeres está bajando, al igual que la participación de los hombres. Es decir que se puede intuir que se trata de un crecimiento en presencia de una reducción del salario medio, con lo cual el efecto "demanda" es menos intenso que el efecto de reducción del costo laboral, en un régimen orientado por beneficio.

Si bien tanto en el caso de la Argentina como en el del Perú se puede afirmar que la ganancia de espacios laborales de las mujeres respecto de una situación anterior de no actividad laboral tiene 
efectos positivos en el ingreso nacional, cabe recordar que las brechas de salario son bastante más elevadas en el caso del Perú, con lo cual este efecto puede tener distinta explicación para los dos países.

Esto quiere decir que, aunque la participación de las mujeres es positiva para el crecimiento (véase el gráfico 5), no siempre es beneficiosa para ellas, dado que la recuperación económica puede no responder a esta concurrencia. Esto puede tener consecuencias en una reducción del salario ofertado, que también es una tendencia en los sectores que experimentan mayor feminización. En los últimos trimestres del período analizado se revierte este proceso. La recesión estuvo acompañada de incrementos en la feminización laboral, dado un mayor desempleo masculino. Es decir que, al igual que en el caso de todos los países analizados, la feminización ocurre como consecuencia de un período recesivo.

Gráfico 5

Resultados de panel: crecimiento y feminización de la oferta laborala (En porcentajes)

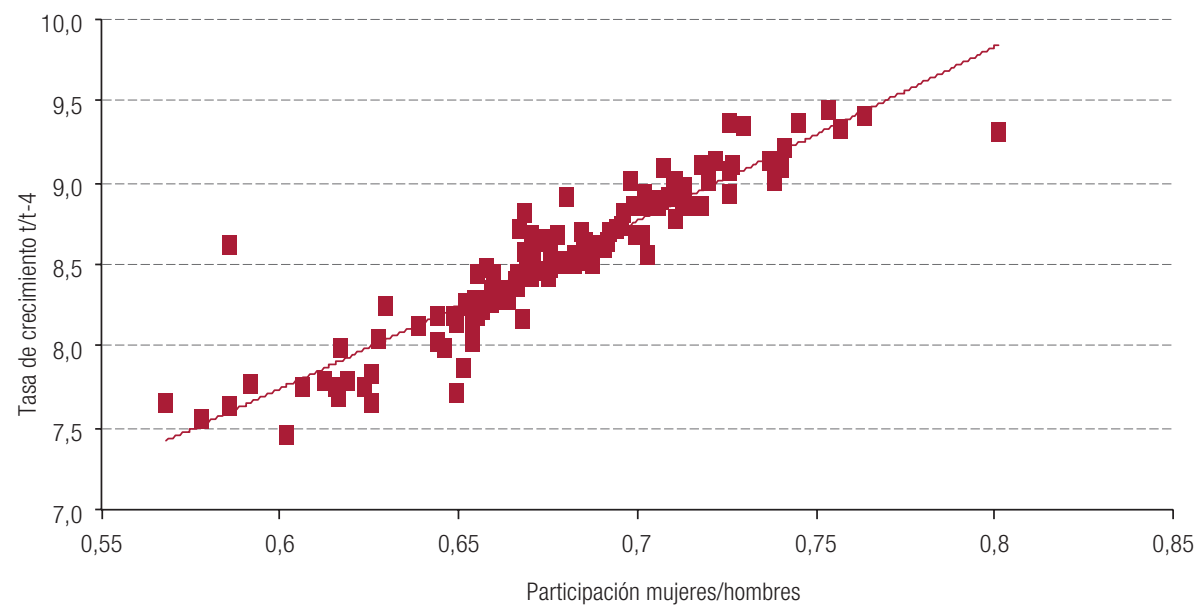

Fuente: Elaboración propia.

a Modelo completo de todos los países de estudio.

Por otra parte, dada la incidencia positiva de la variable de feminización rezagada con un incremento del 19\% del crecimiento del PIB por cada punto de incremento en esta tasa, se debería esperar que esta feminización haya incidido en la recuperación de los períodos posteriores.

De conformidad con los datos observados, en especial para el Ecuador, otra variable de interés es la del crecimiento de la oferta laboral medido en horas, que tiene una relación negativa con el crecimiento económico. El crecimiento de las horas ofertadas por semana está asociado con un decrecimiento del PIB del $8 \%$. Por una parte, la mayor presencia de mujeres frente a su propia situación (su tasa de participación) y la de los hombres es positiva para el crecimiento, pero, por la otra, la intensidad de trabajo (la mayor dedicación) para el mercado (que se intuye que va en detrimento del trabajo doméstico) no lo es. Esto también puede mostrar que un incremento en la productividad (o un mayor esfuerzo de trabajo, en línea con la discusión anterior sobre la elasticidad no compensada de la oferta ante el ingreso laboral propio) puede tener como correlato una reducción de las horas ofertadas, pero elevar la producción y el crecimiento. Del mismo modo, esto puede reflejar un efecto "extra mercado"; es decir, si la tasa de feminización estaba desacelerándose en períodos anteriores (mostrando que las mujeres pudieron salir del mercado para dedicarse al trabajo doméstico como consecuencia de una recesión anterior, por ejemplo), la tasa de crecimiento 
encuentra una recuperación por la vía del "ahorro" de las familias en esta situación ${ }^{13}$. El hecho de que una mayor oferta o intensidad de trabajo de las mujeres se asocie a un decrecimiento del producto puede implicar, en términos de su oferta laboral, que la contribución que hace el trabajo doméstico no remunerado a la recuperación económica es mayor que un incremento de horas con un salario bajo (sesgado en este caso). Esto puede reflejar que el sesgo de género en el mercado laboral es mayor que la diferencia salarial entre hombres y mujeres por características observables (la educación o la experiencia, por ejemplo), y que el salario de una mujer que entra al mercado de trabajo, en promedio, debería ser mayor que lo que el mercado paga en la actualidad.

Cuando se incorporan otros controles sobre el modelo no se modifica la significancia de las variables de interés, pero se explica mejor la tasa de crecimiento. En cuanto a los factores de calificación de la mano de obra, la escolaridad media de la PEA tiene una relación positiva, aunque relativamente baja (cerca del 1\%), con el crecimiento para todas las especificaciones. La significación es igual en magnitud, pero más significativa para la variable rezagada, mostrando también efectos positivos en el plazo medio. En línea con este resultado, la brecha educativa (descrita como la relación entre la escolaridad femenina y masculina de la PEA) presenta coeficientes negativos y significativos para la variable rezagada (22\%), mientras que la contemporánea carece de significación. En virtud de la literatura existente sobre retornos a la educación de hombres y mujeres en la región, la relación entre la escolaridad y el salario es de mayor magnitud para las mujeres que para los hombres, de modo que la eliminación de las brechas podría incidir en un aumento de salarios propios con mayor participación de las mujeres, lo que puede significar un impacto de crecimiento orientado por la demanda en el futuro.

En esta misma línea, un resultado interesante de los modelos desarrollados, que confirma los datos observados, es una relación positiva entre el crecimiento del salario medio y el crecimiento del PIB. Si la tasa de crecimiento del salario se acelera 1 punto, esto resultaría en un incremento del 3\% en la tasa de crecimiento del producto.

En lo que respecta a las variables del contexto macro, el nivel de industrialización tiene una relación negativa con la tasa de crecimiento del PIB, de cerca del 7\%. Esto puede mostrar que los sectores manufactureros han sido poco dinámicos en los últimos años, frente a un proceso de reprimarización y reducción de la diversificación productiva de los países de la región sudamericana; es decir, el crecimiento ha sido promovido por otros sectores y se ha producido a pesar de que la manufactura ha mostrado una tendencia más bien decreciente, en promedio, durante el período analizado. La apertura, en cambio, tiene una relación positiva con el crecimiento en el caso de la variable rezagada (1,8\%), mostrando la orientación exportadora de las economías.

Por último, las dos variables específicas de situación por país también son relevantes en la explicación de la tasa de crecimiento del PIB, de acuerdo con la formulación presentada. La variable de crisis externa, fijada para los dos primeros trimestres de 2003 en la Argentina y el Uruguay, y desde el segundo trimestre de 2009 para todos los países, tiene un efecto negativo de alrededor del 3\% en el crecimiento. A su vez, los cambios de gobierno ocurridos durante el período 2003-2010 están relacionados con un mayor crecimiento en forma positiva y significativa, aunque con un coeficiente bajo (cerca del $1 \%)^{14}$.

\footnotetext{
13 Si bien no existen datos sobre la dedicación horaria al trabajo doméstico para todos los países y en series de tiempo adecuadas para el análisis macroeconómico, la conclusión anterior puede reforzarse con datos de un caso particular: el ecuatoriano. Desde 2003, en las encuestas de empleo del Ecuador existe una batería de preguntas sobre uso del tiempo. Esta información indica que las tasas de crecimiento de la oferta de trabajo de las mujeres tienen un comportamiento inverso a las tasas de crecimiento de la dedicación horaria al trabajo doméstico, en promedio. Por otra parte, estas últimas se comportan en forma similar al crecimiento económico.

${ }^{14}$ El período de análisis coincide con cambios hacia regímenes progresistas en América Latina, caracterizados también por una mayor orientación hacia la demanda del crecimiento y la intervención estatal en las economías. Véase una discusión sobre algunas de las características del período de recuperación latinoamericana y los cambios de timón de estos gobiernos en Machinea y Hopenhayn (2005) y Lustig (2009).
} 
De acuerdo con el marco conceptual planteado, una hipótesis sobre la relación positiva entre feminización y crecimiento indica que la feminización laboral está relacionada con mayores oportunidades de generación de ingresos para las mujeres y con un efecto positivo en la demanda agregada, la inversión y el crecimiento. No obstante, asumiendo que existe una brecha salarial desfavorable a las mujeres, una segunda hipótesis plantea que la entrada de las mujeres al mercado de trabajo reducirá el salario medio, con un efecto en menor costo de producción. Esto implica que la feminización es positiva para el crecimiento, pero este efecto puede acentuarse por la existencia de desigualdades de género, con lo cual estas desigualdades pueden mantenerse como un esquema de ahorro. El resultado podría ser la combinación de ambos efectos. Los resultados anteriores permiten afirmar que durante el período analizado, salvo en el caso brasileño, el primer efecto podría haber dominado al segundo.

\section{Conclusiones y reflexiones finales}

A partir de los resultados planteados es posible presentar algunas conclusiones básicas. En relación con la oferta laboral, se encuentra que la selección negativa, es decir, la no inclusión de personas que podrían estar en el mercado laboral y no están (en este caso, las mujeres), se mantiene casi intacta durante siete años de estudio en los países, e incluso tiende a elevarse. Una revisión más detallada muestra que esta selección se da más en los sectores de empleo pleno y en relación de dependencia (que son empleos mejor pagados y usualmente más estables). Esto ocurre a pesar de que los países presentan altas tasas de crecimiento hacia mediados de la década de 2000 y hasta 2008, lo que continúa hacia 2009. Se podría intuir que se ha tratado de modalidades de crecimiento que han privilegiado la intensificación del uso de la mano de obra antes que la apertura a la generación de oportunidades de empleo, aunque en algunos casos (por ejemplo, el Brasil y el Uruguay), los salarios medios no se han incrementado acorde al crecimiento de la economía. En esta línea, los sectores con mayor generación de empleo para las mujeres han sido los de servicios, que tienen un retorno negativo en términos de salario.

Las estimaciones de oferta laboral para los países estudiados también permiten señalar que existen tres grandes tendencias. La primera es un decrecimiento del sesgo por selección, tanto para hombres como para mujeres en el Ecuador y el Perú, y solamente para las mujeres en la Argentina y el Uruguay. De hecho, en el Ecuador y el Uruguay, el sesgo para hombres es cercano a cero al final de 2009. Este decrecimiento general se acompaña con cierta convergencia entre ambos grupos, salvo para el caso de la Argentina.

En el Brasil ocurre algo distinto, dado que se registran valores crecientes de selección global, debido a un coeficiente theta muy elevado a partir de 2007, lo que potencia la selección lambda, que es similar a la de otros países. Se debe indicar que este incremento es parecido para hombres y mujeres. No obstante, también existe convergencia hacia el último año.

La segunda tendencia es un incremento de la feminización laboral debido a un aumento en la participación femenina en todos los casos y a una reducción de la oferta masculina en la Argentina y el Ecuador en 2009. En estos últimos casos podría haber una relación con la reciente crisis financiera, una de cuyas consecuencias fue la reducción de la PEA en ambos países. Este incremento en la participación va acompañado, en la mayoría de los casos, de reducciones en la intensidad del trabajo (horas por semana), lo que permite intuir que el subempleo sigue siendo la principal opción de trabajo para las mujeres.

Al respecto, la tercera tendencia observada es que la participación laboral femenina tiene igual tendencia que el crecimiento económico, pero sus fluctuaciones son contracíclicas al igual 
que su tasa de crecimiento. Es decir, la desaceleración del crecimiento de esta oferta laboral puede estar mostrando una probable sustitución de actividades en el hogar que permiten algún nivel de recuperación económica, aunque la creciente presencia de mujeres (en relación con su condición de no entrada) es positiva e importante para el crecimiento económico y para su situación individual.

En consecuencia, la inserción en el mercado de trabajo, si bien es creciente, no asegura la incorporación a un empleo, en especial en condiciones adecuadas. Por lo general, persisten niveles de desempleo, basados en una baja capacidad de absorción de mano de obra, pero también de subempleo, basados en la limitada generación de empleo productivo. Las deficiencias en la estructura de empleo se manifiestan tanto por la persistencia de altas tasas de desempleo como por las distintas formas de subempleo. Estos resultados coinciden con mucha de la literatura empírica referida a la situación laboral de las mujeres en América Latina, en especial en los últimos años y durante la recesión de 2008 y 2009.

Sin duda, la entrada de las mujeres al mercado laboral supone una mejor situación individual y familiar, y, de acuerdo con lo analizado en esta investigación, una mejor situación de la economía en general. La feminización laboral dada por la reducción de las brechas de participación entre hombres y mujeres genera incrementos del PIB tanto en valores contemporáneos como en términos rezagados, y también muestra un potencial efecto positivo de mediano plazo. No obstante, el incremento de las horas trabajadas (intensidad del trabajo) tiene una relación inversa con el crecimiento, lo que da cuenta de un posible impacto negativo de la reducción del tiempo dedicado a los cuidados sin que haya sustitución por servicios públicos o privados, o corresponsabilidad de otros miembros del hogar. Para verificar esta intuición es necesario realizar un análisis sobre el trabajo doméstico y el ahorro de los hogares frente a la oferta laboral femenina, en línea con lo presentado en el marco teórico.

Otra variable relevante que incentiva el crecimiento económico es el crecimiento (rezagado) del salario medio. Esto muestra la factibilidad de un crecimiento orientado por la demanda, pero además está en línea con lo anterior, dado que si bien un salario bajo en el período actual puede incentivar el crecimiento, el salario debe evolucionar positivamente para que este crecimiento se mantenga.

En consecuencia, tanto la "selección" negativa como la diferencia salarial, fruto de la desigualdad horizontal, pueden impactar en forma positiva en el crecimiento en lo inmediato por dos vías: la reducción de costos laborales y el ahorro de las familias en caso de que las mujeres dediquen más tiempo al trabajo doméstico o se ocupen en tareas menos estables.

Si las políticas económicas aplicadas y la orientación hacia las exportaciones de muchas de las economías analizadas ponen énfasis en las ganancias de corto plazo, la situación de discriminación y brechas salariales puede continuar apoyando este tipo de modalidad de crecimiento, de modo que es necesario pensar en políticas que apunten a cambios en la estructura y orientación productiva, y que permitan que los factores de demanda conduzcan a un crecimiento más basado en la equidad, con la equidad como una meta per se (no solo "complementaria" al crecimiento) de estas políticas económicas.

En cuanto al nivel meso del mercado y la política laboral, a partir del análisis anterior, es posible mencionar algunos elementos clave para la reflexión sobre las políticas públicas orientadas a mejorar la participación laboral de las mujeres y sus potenciales efectos, en función del círculo virtuoso feminización-incremento de la demanda-crecimiento.

El primer elemento tiene que ver con la organización del trabajo, incorporando en la definición de trabajo el continuo cuidado-mercado, con la finalidad de que el régimen laboral funcione de acuerdo con las exigencias del cuidado y del sistema económico. Esto se relaciona con la ruptura de importantes barreras para el ingreso al mercado, que combinan protección social y una política laboral y económica que asegure recursos permanentes para el sostenimiento de sistemas integrados de servicios. En el caso de la política laboral, se requiere que el régimen de trabajo incorpore las 
necesidades del ciclo de vida de las familias, y la aplicación de la normativa familiar en igual proporción para los hombres en el nivel general, de tal forma que las mujeres no sean vistas por los empleadores como trabajadoras costosas, y que su mayor participación horaria en el trabajo de mercado tenga como correlato un impacto positivo en la demanda agregada, la productividad y el crecimiento.

Un segundo elemento está relacionado con el proceso de formación y adaptación para el trabajo, y con el nivel de redes e información al que acceden las mujeres sobre demanda de trabajo, salarios de mercado y procesos de selección, así como la regulación e inspectoría del trabajo para asegurar la reducción de la segregación y las brechas salariales. La incorporación de elementos que den cuenta de la igualdad entre hombres y mujeres, la no discriminación, la no segregación y la corresponsabilidad social sobre el cuidado deben profundizarse en la normativa y en los procesos de control estatal, así como en las agendas de las organizaciones de trabajadores y trabajadoras.

Finalmente, desde el punto de vista metodológico, la investigación deja algunas reflexiones en cuanto a necesidades de revisiones y análisis futuros, que puedan ayudar a profundizar la relación entre el crecimiento y la desigualdad de género (y la desigualdad en general). Si bien la medición de los valores transmitidos a través de las desigualdades de género en el ámbito reproductivo no es posible con la información disponible, mediante el análisis de la parte visible de la economía se puede afirmar que el ámbito reproductivo tiene incidencia en el régimen económico laboral y ello, a su vez, incide en la economía más ampliamente considerada, a través de la consideración de la oferta laboral como el punto de unión entre la economía mercantil y el ámbito doméstico, fruto de un proceso de conflicto y generadora de fuerza de trabajo, que ha sido el camino metodológico seguido en esta investigación. No obstante, se hace necesario incluir en el análisis macro mejores y más completos indicadores de desarrollo (además del crecimiento) y nuevas mediciones de la desigualdad de género. También es necesaria la reflexión sobre modelos teóricos más acordes a la concepción de una economía integrada. La endogeneización de la oferta laboral debe ser reconsiderada asumiendo que no se trata (únicamente) de un proceso de elección entre usos del tiempo o entre ahorrar y consumir, sino que se basa en procesos complejos que dependen de la estructura de la economía, pero también de ordenamientos sociales y sistemas de conflictos.

\section{Bibliografía}

Agarwal, B. (1997), "'Bargaining' and gender relations: within and beyond the household", Feminist Economics, vol. 3, No 1, Taylor \& Francis.

Aguero, J. y M. Marks (2008), "Motherhood and female labor participation: evidence from infertility shocks", American Economic Review, vol. 98, № 2, Nashville, Tennessee, American Economic Association.

Benería, L. (2008), "The crisis of care, international migration, and public policy", Feminist Economics, vol. 14, № 3, Taylor \& Francis.

(2005), "Vinculaciones entre lo global y lo local. Patrones de empleo, género e informalización”, Género, desarrollo y globalización: por una ciencia económica para todas las personas, Barcelona, Hacer.

(2003), Gender, Development and Globalization: Economics as if all People Mattered, Nueva York, Routledge.

Berger, S. y H. Szretter (2002), "Costos laborales de hombres y mujeres. El caso de Argentina", Cuestionando un mito: costos laborales de hombres y mujeres en América Latina, L. Abramo y R. Todaro (eds.), Lima, Organización Regional de la OIT para América Latina y el Caribe.

Berik, G. e Y. Rodgers (2008), "Engendering development strategies and macroeconomic policies: what's sound and sensible?", Social Justice and Gender Equality: Rethinking Development Strategies and Macroeconomic Policies, G. Berik, Y. Rodgers y A. Zammit (eds.), Nueva York, Routledge.

Berik, G., Y. Rodgers y S. Seguino (2009), "Feminist economics of inequality, development and growth", Feminist Economics, vol. 15, № 3, Taylor \& Francis.

Berndt, E.R. (1990), The Practice of Econometrics: Classic and Contemporary, Reading, Addison Wesley.

Bhaduri, A. (2007), "On the dynamics of profit-led and wage-led growth", Cambridge Journal of Economics, vol. $32, N^{\circ} 1$. 
Blackden, M. y C. Bhanu (1999), "Gender, growth, and poverty reduction: special program of assistance for Africa, 1998 status report on poverty in Sub-Saharan Africa", Technical Paper, № 428, Washington, D.C., Banco Mundial.

Blackden, M. y otros (2007), "Gender and growth in Africa: evidence and issues", Advancing Development: Core Themes in Global Development, G. Mavrotas y A. Shorrocks (eds.), Palgrave Macmillan.

Blau, F., M. Ferber y A. Winkler (2002), The Economics of Women, Men and Work, Upper Saddle River, Prentice Hall.

Blecker, R. y S. Seguino (2002), "Macroeconomic effects of reducing gender wage inequality in an exportoriented semi industrialized economy", Review of Development Economics, vol. 6, № 1, Wiley.

Blundell, R., T. MaCurdy y C. Meghir (2007), "Labor supply models: unobserved heterogeneity, nonparticipation and dynamics. Specification in Labor Supply and Consumption Models", Londres, University College London.

Braunstein, E. (2008), "The feminist political economy of the rent-seeking society: an investigation of gender inequality and economic growth", Journal of Economic Issues, vol. 42, $N^{\circ} 4$, Taylor \& Francis.

Carrasco, C. (2003), "Tiempos, trabajos y organización social: reflexiones en torno al mercado laboral femenino", Mujeres y economía, Barcelona, Icaria.

(2001), "La sostenibilidad de la vida humana: ¿un asunto de mujeres?", Mientras Tanto, № 82, Barcelona, Icaria Editorial.

Cavalcanti, T. y J. Tavares (2007), "The output cost of gender discrimination: a model-based macroeconomic estimate", CEPR Discussion Paper, Nㅜ 6477, Londres, Centro de Investigación sobre Políticas Económicas.

CEPAL (Comisión Económica para América Latina y el Caribe) (2009a), "Anexo estadístico", Panorama Social de América Latina, 2009 (LC/G.2423-P), Santiago.

(2009b), "América Latina y el Caribe: series históricas de estadísticas económicas 1950-2008", Cuadernos Estadísticos, № 37 (LC/G.2415-P), Santiago.

Cerrutti, M. (2000), "Economic reform, structural adjustment and female labor force participation in Buenos Aires, Argentina", World Development, vol. 28, № 5, Amsterdam, Elsevier.

Contreras, J., A. Armas y A. Vásconez (2008), La economía del cuidado, el trabajo no remunerado y remunerado en Ecuador, Quito.

Cruces, G. y S. Galiani (2007), "Fertility and female labor supply in Latin America: new causal evidence", Labour Economics, vol. 14, № 3, Amsterdam, Elsevier.

Darity, W. (1995), "The formal structure of a gender-segregated low-income economy", World Development, vol. 23, No 11, Amsterdam, Elsevier.

Dollar, D. y R. Gatti (1999), "Gender inequality, income, and growth: are good times good for women?", Policy Research Report on Gender and Development. Working Paper Series, № 1, Washington, D.C., Banco Mundial.

Doss, C. y M. Morris (2001), "How does gender affect the adoption of agricultural innovations?: The case of improved maize technology in Ghana", Agricultural Economics, vol. 25, № 1, Amsterdam, Elsevier.

Dutt, A.K. (1990), Growth, Distribution and Uneven Development, Cambridge, Cambridge University Press.

Elson, D. (1999), "Labor markets as gendered institutions: equality, efficiency and empowerment issues", World Development, vol. 27, №3, Amsterdam, Elsevier.

(1995a), "Gender awareness in modeling structural adjustment", World Development, vol. 23, № 11, Amsterdam, Elsevier. (1995b), Male Bias in the Development Process, Nueva York, Manchester University Press.

Erturk, K. y N. Cagatay (1995), "Macroeconomic consequences of cyclical and secular changes in feminization: an experiment at gendered macromodeling", World Development, vol. 23, № 11, Amsterdam, Elsevier.

Erturk, K. y W. Darity (2000), "Secular changes in the gender composition of employment and growth dynamics in the North and the South", World Development, vol. 28, № 7, Amsterdam, Elsevier.

Espino, A. y P. Azar (2005), "Changes in economic policy regimes in Uruguay from a gender perspective (1930-2000)", GEM-IWG Working Paper, Nº6-05, Utah, Universidad de Utah.

Espino, A., M. Leites y A. Machado (2009), "El aumento en la oferta laboral de las mujeres casadas en Uruguay", Desarrollo y Sociedad, № 64, Universidad de Los Andes.

Esquivel, V. (2005), "Antecedentes nacionales e internacionales de estudios sobre diferenciales de ingresos laborales por género", Buenos Aires, Ministerio de Trabajo, Empleo y Seguridad Social.

Esteve-Volart, B. (2004), "Gender discrimination and growth: theory and evidence from India", Development Economics Papers, $N^{\circ} 42$, Londres, London School of Economics. 
Heckman, J. (1993), "What has been learned about labor supply in the past twenty years?", American Economic Review, vol. 83, № 1, Nashville, Tennessee, American Economic Association.

_ (1979), "Sample bias as a specification error", Econometrica, vol. 47, № 1, Nueva York, The Econometric Society.

_ (1976), "The common structure of statistical models of truncation, sample selection and limited dependent variables and a simple estimator for such models", Annals of Economic and Social Measurement, vol. 5, $N^{\circ} 4$, Cambridge, Massachusetts, National Bureau of Economic Research.

Killingsworth, M. (1983), Labor Supply, Nueva York, Cambridge University Press.

King, E. y M. Porter (2010), "Fertility and women's labour force participation in developing countries", documento presentado en la Population and Poverty Research Network Conference, Ciudad del Cabo.

Klasen, S. (2006), "Pro-poor growth and gender inequality", Discussion Paper, № 151, Ibero-America Institute for Economic Research.

(2002), "Low schooling for girls, slower growth for all? Cross-country evidence on the effect of gender inequality in education on economic development", World Bank Economic Review, vol. 16, № 3, Washington, D.C., Banco Mundial.

Klasen, S. y F. Lamanna (2009), "The impact of gender inequality in education and employment on economic growth: new evidence for a panel of countries", Feminist Economics, vol. 15, № 3, Taylor \& Francis.

Kucera, D. (2002), "The effects of wealth and gender inequality on economic growth: a survey of recent empirical studies, Discussion Paper, № 136, Ginebra, Instituto Internacional de Estudios Laborales.

Lo Vuolo, R. (2009), Distribución y crecimiento, una controversia persistente, Buenos Aires, Miño y Dávila.

Lustig, N. (2009), "La pobreza y la desigualdad en América Latina y los gobiernos de izquierda", Cuadernos, № 7, Ciudad de México, Consejo Mexicano de Asuntos Internacionales.

Machinea, J.L. y M. Hopenhayn (2005), "La esquiva equidad en el desarrollo latinoamericano", serie Informes y Estudios Especiales, № 14 (LC/L.2414-P), Santiago, Comisión Económica para América Latina y el Caribe (CEPAL).

Martín, F. (2008), Convergencia en renta per cápita entre las comunidades autónomas españolas (19552004): una aplicación basada en métodos de panel dinámico, Madrid, Fundación de las Cajas de Ahorro.

Novick, M. y S. Rojo (2009), "El trabajo femenino en la post convertibilidad: Argentina 2003-2007”, Santiago.

Pérez, A. (2005), "Economía de género y economía feminista: conciliación o ruptura?", Revista Venezolana de Estudios de la Mujer, vol. 10, Caracas.

Picchio, A. (2003), "A macroeconomic approach to an extended styard of living", Unpaid Work and the Economy. A Gender Analysis of the Standards of Living, A. Picchio (ed.), Londres, Routledge.

Rodríguez, C., V. Esquivel y A. Espino (2012), "Crisis, regímenes económicos e impactos de género en América Latina", La economía feminista desde América Latina: una hoja de ruta sobre los debates actuales en la región, V. Esquivel (ed.), Santo Domingo.

Ros, J. (2004), La teoría del desarrollo y la economía del crecimiento, Ciudad de México, Fondo de Cultura Económica.

Rubery, J. (1988), Women and Recessions, Nueva York, Routledge.

Seguino, S. (2010), "Gender, distribution, and balance of payments constrained growth in developing countries", Review of Political Economy, vol. 22, № 3, Taylor \& Francis.

(2000), "Gender inequality and economic growth: a cross-country analysis", World Development, vol. 28, $N^{\circ} 7$, Amsterdam, Elsevier.

Sen, A. (2000), "Género y conflictos cooperativos", Cambios sociales, económicos y culturales: un nuevo saber, los estudios de mujeres, M. Navarro y C. Stimpson (comps.), Ciudad de México, Fondo de Cultura Económica.

Stotsky, J. (2006), "Gender and its relevance to macroeconomic policy: a survey", IMF Working Paper, $N^{\circ}$ 06/233, Washington, D.C., Fondo Monetario Internacional.

Todaro, R. y S. Yáñez (2004), El trabajo se transforma. Relaciones de producción y relaciones de género, Santiago, Ediciones CEM.

Vásconez, A. (2012), "Mujeres, hombres y economías latinoamericanas: un análisis de dimensiones y políticas", La economía feminista desde América Latina: una hoja de ruta sobre los debates actuales en la región, V. Esquivel (ed.), Santo Domingo. 


\section{Anexo A1}

Cuadro A1.1

Test de especificación para efectos fijos: variable de feminización

\begin{tabular}{lcccc}
\hline Especificación 1 & $\begin{array}{c}(\mathrm{b}) \\
\text { FIXED_2 }\end{array}$ & $\begin{array}{c}(\mathrm{B}) \\
\text { RAMDOM_2 }\end{array}$ & $\begin{array}{c}(\mathrm{b}-\mathrm{B}) \\
\text { Difference }\end{array}$ & $\begin{array}{c}\text { sqrt(diag(V_b-V_B) } \\
\text { S.E. }\end{array}$ \\
\hline inversion ib & $-0,1686565$ & 0,0573637 & $-0,2260203$ & 0,0992541 \\
\hline L.inversi ib & 0,0303124 & $-0,1473261$ & 0,1776385 & 0,0886562 \\
\hline L2.invers ib & 0,0288376 & $-0,2557388$ & 0,2845764 & 0,1338661 \\
\hline feminizacion & 0,7887797 & 0,0185267 & 0,770253 & 0,4044201 \\
\hline L.feminiza n & $-0,3723222$ & $-0,3534988$ & $-0,0188234$ &. \\
\hline L2.feminiz n & 0,0647415 & $-0,1011472$ & 0,1658887 & 0,0911338 \\
\hline escolarida a & $-0,0059632$ & $-0,0063436$ & 0,0003804 & 0,0040306 \\
\hline fem_argent a & $-1,341968$ & $-0,0225667$ & $-1,319401$ & 0,8209595 \\
\hline fem_brasil & $-0,5604077$ & $-0,1900654$ & $-0,3703423$ & 0,595131 \\
\hline fem_ecuador & $-0,9882586$ & $-0,129229$ & $-0,8590296$ & 0,4538183 \\
\hline fem_peru & $-0,3081725$ & $-0,0662077$ & $-0,2419648$ & 0,6024027 \\
\hline d_gobierno & 0,0023519 & 0,0104388 & $-0,008087$ & 0,0043851
\end{tabular}

b: consistente bajo las hipótesis nula $(\mathrm{Ho})$ y alternativa (Ha). Coeficiente estimado por efectos fijos. Test B: inconsistente bajo Ha, eficiente bajo Ho. Coeficiente estimado por efectos fijos.

Ho: la diferencia de coeficientes no es sistemática

Chi cuadrado con 12 grados de libertad=(b-B)'[N_b-V_B $\left.)^{\wedge}(-1)\right](b-B)=5,13$

Prob $>$ Chi cuadrado $=0,9536$

\begin{tabular}{lcccc}
\hline Especificación 2 & $\begin{array}{c}\text { (b) } \\
\text { FIXED_3 }\end{array}$ & $\begin{array}{c}(\mathrm{B}) \\
\text { RAMDOM_3 }\end{array}$ & $\begin{array}{c}(\mathrm{b}-\mathrm{B}) \\
\text { Difference }\end{array}$ & $\begin{array}{c}\text { sqrt(diag(V_b-V_B })) \\
\text { S.E. }\end{array}$ \\
\hline inversion ib & 0,0947441 & 0,1097889 & $-0,0150449$ & 0,1125835 \\
\hline L.inversi ib & 0,0577002 & 0,0185697 & 0,0391305 & 0,1330804 \\
\hline L2.invers ib & 0,1269483 & 0,1319894 & $-0,0050411$ & 0,110423 \\
\hline feminizacion & 0,0666373 & 0,1137812 & $-0,0471439$ & 0,102343 \\
\hline L.feminiza n & $-0,3334827$ & $-0,3143287$ & $-0,019154$ & 0,114324 \\
\hline L2.feminiz n & $-0,0687099$ & 0,2736242 & $-0,3423342$ & 0,0847786 \\
\hline crec_horas_m & $-0,0658539$ & $-0,106271$ & 0,0404172 & 0,0523413 \\
\hline L.crec_hor m & $-0,0926459$ & $-0,1033992$ & 0,0107533 & 0,0267719 \\
\hline crec_salar 4 4 & 0,0039381 & 0,0184609 & $-0,0145228$ & 0,0045453 \\
\hline L.crec_sal 4 & $-0,0105544$ & $-0,0102478$ & $-0,0003066$ & 0,0110023 \\
\hline L2.crec_sa 4 & 0,0325524 & 0,0144698 & 0,0180825 & 0,0047757 \\
\hline apertura & 0,0059945 & $-0,0042663$ & 0,0102608 & 0,0035646 \\
\hline L.apertura & 0,0356175 & 0,0091102 & 0,0265073 & 0,0065033 \\
\hline L2.apertura & 0,0508193 & $-0,007941$ & 0,0587603 & 0,0252948 \\
\hline manufactura & 0,5873649 & 0,2079639 & 0,379401 & 0,2420066 \\
\hline L.manufact a & $-0,2910921$ & $-0,1930398$ & $-0,0980523$ & 0,011293 \\
\hline L2.manufac a & 0,3245035 & 0,0669439 & 0,2575596 & 0,1690496 \\
\hline brecha_esc a & 0,3946982 & 0,2321393 & 0,1625589 & 0,0861091 \\
\hline escolarida a & $-0,025813$ & $-0,0050629$ & $-0,0207501$ & 0,093747 \\
\hline
\end{tabular}


b: consistente bajo las hipótesis nula (Ho) y alternativa (Ha). Coeficiente estimado por efectos fijos. Test B: inconsistente bajo Ha, eficiente bajo Ho. Coeficiente estimado por efectos fijos.

Ho: la diferencia de coeficientes no es sistemática

Chi cuadrado con 20 grados de libertad=(b-B)'[N_b-V_B $\left.)^{\wedge}(-1)\right](b-B)=13,45$

Prob $>$ Chi cuadrado $=0,2674$

\begin{tabular}{|c|c|c|c|c|}
\hline Especificación 3 & $\begin{array}{c}\text { (b) } \\
\text { FIXED_4 }\end{array}$ & $\begin{array}{c}\text { (B) } \\
\text { RAMDOM_4 }\end{array}$ & $\begin{array}{c}(\mathrm{b}-\mathrm{B}) \\
\text { Difference }\end{array}$ & $\begin{array}{c}\text { sqrt(diag(V_b-V_B })) \\
\text { S.E. }\end{array}$ \\
\hline inversion ib & $-0,3105985$ & $-0,0250665$ & $-0,285532$ & 0,1501545 \\
\hline L.inversi ib & 0,4257956 & 0,1472383 & 0,2785573 & 0,1536835 \\
\hline 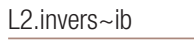 & 0,3680402 & 0,0935648 & 0,2744754 & 0,1559726 \\
\hline feminizacion & 1,123705 & 0,2364684 & 0,8872363 & 0,550181 \\
\hline L.feminiza n & $-0,1357549$ & $-0,1381812$ & 0,0024263 & 0,0200397 \\
\hline L2.feminiz n & 0,0429889 & $-0,1774335$ & 0,2204224 & 0,1225627 \\
\hline crec_salar 4 & 0,0042054 & $-0,0005276$ & 0,0047329 & 0,0059859 \\
\hline L.crec_sal 4 & $-0,0096526$ & 0,0064797 & $-0,0161323$ & 0,0122084 \\
\hline L2.crec_sa 4 & 0,0282299 & 0,0286311 & $-0,0004012$ & 0,0052577 \\
\hline apertura & 0,0005758 & 0,0046368 & $-0,0040611$ & 0,0040646 \\
\hline L.apertura & 0,0367211 & 0,0329127 & 0,0038084 & 0,0045161 \\
\hline L2.apertura & 0,0681435 & 0,052462 & 0,0156815 & 0,0125536 \\
\hline manufactura & 0,7853062 & 0,6188142 & 0,166492 & 0,2389519 \\
\hline L.manufact a & $-0,3755361$ & $-0,3216545$ & $-0,0538816$ & 0,0835781 \\
\hline L2. manufac a & 0,4140829 & 0,2811619 & 0,132921 & 0,0803168 \\
\hline brecha_esc a & 0,1563221 & 0,3115227 & $-0,1552006$ & 0,1371424 \\
\hline escolarida a & $-0,0194555$ & $-0,0207719$ & 0,0013165 & 0,0042069 \\
\hline fem_argent a & $-1,571086$ & $-0,0072999$ & $-1,563786$ & 0,9585457 \\
\hline fem_brasil & $-0,696639$ & $-0,0361616$ & $-0,6604774$ & 0,9805168 \\
\hline fem_ecuador & $-1,072292$ & $-0,1328174$ & $-0,9394745$ & 0,5912154 \\
\hline fem_peru & $-0,3028901$ & $-0,0071759$ & $-0,2957141$ & 0,6760553 \\
\hline d_gobierno & $-0,0042728$ & 0,0081865 & $-0,0124593$ & 0,0065887 \\
\hline d_recesion & $-0,0342451$ & $-0,0314171$ & $-0,002828$ & 0,0034887 \\
\hline
\end{tabular}

b: consistente bajo las hipótesis nula (Ho) y alternativa (Ha). Coeficiente estimado por efectos fijos.

B: inconsistente bajo Ha, eficiente bajo Ho. Coeficiente estimado por efectos fijos.

Test

Ho: la diferencia de coeficientes no es sistemática

Chi cuadrado con 23 grados de libertad=(b-B)' $\left[\left(\mathrm{N} \_b-V \_B\right) \wedge(-1)\right](b-B)=4,19$

Prob $>$ Chi cuadrado $=0,96765$

Fuente: Elaboración propia. 


\section{Anexo A2}

Cuadro A2.1

Endogeneidad y formas reducidas para verificación de instrumentos en variables de interés

\begin{tabular}{|c|c|c|c|}
\hline Variable & Coeficiente & $\mathrm{F}$ & Prob $>F$ \\
\hline \multirow[t]{2}{*}{ FEM (t-1) } & $0,631^{\star \star \star}$ & 77,79 & 0,000 \\
\hline & 0,071 & & \\
\hline \multirow[t]{2}{*}{ FEM (t-1) } & $0,287^{* * *}$ & 46,09 & 0,001 \\
\hline & 0,042 & & \\
\hline Variable & Coeficiente & $\mathrm{F}$ & Prob $>\mathrm{F}$ \\
\hline \multirow[t]{2}{*}{ Dif_FEM (t-1) } & $0,796^{\star \star \star}$ & 14,50 & 0,036 \\
\hline & 0,038 & & \\
\hline \multirow[t]{2}{*}{ Dif_FEM (t-1) } & $0,447^{* * *}$ & 11,34 & 0,025 \\
\hline & 0,099 & & \\
\hline
\end{tabular}

Fuente: Elaboración propia.

Nota: * significativo al 90\%; ${ }^{* \star}$ significativo al $95 \%$; ${ }^{* \star \star}$ significativo al $99 \%$.

\section{Anexo A3}

\section{Cuadro A3.1}

Resultados de modelos de panel

Variable de interés: feminización de la oferta laboral

\begin{tabular}{|c|c|c|c|c|c|c|}
\hline & \multicolumn{3}{|c|}{ Mínimos cuadrados (pool de datos) } & \multicolumn{3}{|c|}{$\begin{array}{l}\text { Panel autorregresivo corregido por } \\
\text { endogeneidad var. independientes }\end{array}$} \\
\hline & Modelo 1 & Modelo 2 & Modelo 3 & Modelo 1 & Modelo 2 & Modelo 3 \\
\hline \multicolumn{7}{|c|}{ Variable dependiente: crecimiento PIB t/t-4 } \\
\hline \multirow[t]{2}{*}{ Crecimiento PIB(t-1), I(2) } & & & & $0,607^{\star \star *}$ & 0,638 *** & $0,607^{* * *}$ \\
\hline & & & & 0,076 & 0,071 & 0,099 \\
\hline \multicolumn{7}{|l|}{ Crecimiento PIB (t-2) } \\
\hline \multirow[t]{2}{*}{ Inversión/PIB } & $0,131^{\star}$ & 0,169 *** & 0,186 ** & $0,092^{\star \star \star}$ & 0,077 & 0,134 \\
\hline & 0,067 & 0,051 & 0,078 & 0,028 & 0,112 & 0,091 \\
\hline \multirow[t]{2}{*}{ Inversión rezagada (t-1) } & & & & $0,137^{* \star *}$ & $0,121^{* * *}$ & $0,129 * * *$ \\
\hline & & & & 0,030 & 0,034 & 0,032 \\
\hline \multicolumn{7}{|l|}{ Inversión rezagada (t-2) } \\
\hline \multirow[t]{2}{*}{ Feminización de la oferta laboral } & $0,040^{\star *}$ & $0,047^{* *}$ & 0,031 * & $0,057^{* *}$ & $0,055^{\star *}$ & 0,059 ** \\
\hline & 0,019 & 0,022 & 0,020 & 0,024 & 0,025 & 0,026 \\
\hline \multirow[t]{2}{*}{ Feminización (t-1) } & & & & $0,073^{*}$ & 0,079 ** & 0,077 ** \\
\hline & & & & 0,040 & 0,039 & 0,037 \\
\hline \multicolumn{7}{|l|}{ Feminización (t-2) } \\
\hline \multirow[t]{2}{*}{ Capital humano PEA } & 0,006 * & $0,005^{* *}$ & $0,007^{\text {*** }}$ & $0,008^{* * *}$ & 0,009 * & 0,008 * \\
\hline & 0,003 & 0,003 & 0,002 & 0,003 & 0,005 & 0,004 \\
\hline \multirow[t]{2}{*}{ Capital humano PEA (I_t-1) } & & & & $0,008^{* * *}$ & $0,008^{* *}$ & 0,010 *** \\
\hline & & & & 0,003 & 0,004 & 0,003 \\
\hline
\end{tabular}


Cuadro A3.1 (conclusión)

\begin{tabular}{|c|c|c|c|c|c|c|}
\hline & \multicolumn{3}{|c|}{ Mínimos cuadrados (poo/ de datos) } & \multicolumn{3}{|c|}{$\begin{array}{l}\text { Panel autorregresivo corregido por } \\
\text { endogeneidad var. independientes }\end{array}$} \\
\hline & Modelo 1 & Modelo 2 & Modelo 3 & Modelo 1 & Modelo 2 & Modelo 3 \\
\hline \multirow[t]{2}{*}{ Capital humano PEA (t-2) } & & & & & $0,003^{* \star \star}$ & 0,004 ** \\
\hline & & & & & 0,001 & 0,002 \\
\hline \multirow[t]{2}{*}{ Feminización_Argentina } & $0,015^{* * *}$ & $0,013^{* \star *}$ & 0,010 *** & & 0,049 *** & $0,047^{* \star *}$ \\
\hline & 0,001 & 0,002 & 0,002 & & 0,016 & 0,013 \\
\hline \multirow[t]{2}{*}{ Feminización_Brasil } & 0,099 *** & $-0,088$ *** & 0,070 ** & & $-0,013$ & $-0,014$ \\
\hline & 0,032 & 0,030 & 0,031 & & 0,016 & 0,028 \\
\hline \multirow[t]{2}{*}{ Feminización_Ecuador } & $-0,057^{* *}$ & $-0,064^{\star \star \star}$ & $-0,056$ ** & & $-0,004$ & $-0,008$ \\
\hline & 0,024 & 0,020 & 0,022 & & 0,008 & 0,013 \\
\hline \multirow[t]{2}{*}{ Feminización_Perú } & $-0,029$ & $-0,035^{*}$ & $-0,031$ & & 0,021 * & 0,024 * \\
\hline & 0,022 & 0,020 & 0,024 & & 0,013 & 0,013 \\
\hline \multirow[t]{2}{*}{ Crecimiento oferta horas } & & 0,001 & & & $-0,063^{\star *}$ & $-0,081^{* \star *}$ \\
\hline & & 0,045 & & & 0,031 & 0,022 \\
\hline \multirow[t]{2}{*}{ Crec_oferta_(t-1) } & & & & & 0,030 & $0,077^{*}$ \\
\hline & & & & & 0,050 & 0,042 \\
\hline \multicolumn{7}{|l|}{ Crec_oferta_(t-2) } \\
\hline \multirow[t]{2}{*}{ Crec_salario_medio } & & & & & $0,033^{* * *}$ & $0,027^{* * *}$ \\
\hline & & & & & 0,009 & 0,011 \\
\hline \multirow[t]{2}{*}{ Crec_salario_medio(t-1) } & & $0,025^{* * \star}$ & $0,021^{* \star *}$ & & $-0,017^{\star \star \star}$ & 0,009 \\
\hline & & 0,009 & 0,008 & & 0,005 & 0,016 \\
\hline \multicolumn{7}{|l|}{ Crec_salario_medio(t-2) } \\
\hline \multirow[t]{2}{*}{ Brecha escolaridad } & & $-0,172$ & $-0,151^{*}$ & & 0,325 & 0,281 \\
\hline & & 0,747 & 0,096 & & 0,322 & 0,218 \\
\hline \multirow[t]{2}{*}{ Brecha escolaridad (I_t-1) } & & & & & $-0,226$ * & $-0,254$ * \\
\hline & & & & & 0,130 & 0,146 \\
\hline \multirow[t]{2}{*}{ Apertura económica } & & 0,004 ** & 0,005 & & $-0,003^{* *}$ & 0,003 \\
\hline & & 0,002 & 0,176 & & 0,001 & 0,002 \\
\hline \multirow[t]{2}{*}{ Apertura (t-1) } & & & & & $0,018^{* \star *}$ & $0,018^{* * *}$ \\
\hline & & & & & 0,005 & 0,004 \\
\hline \multirow[t]{2}{*}{ Apertura (t-2) } & & & & & $-0,020 * \star *$ & $-0,007$ \\
\hline & & & & & 0,004 & 0,011 \\
\hline \multirow[t]{2}{*}{ Industrialización } & & $-0,065^{\star *}$ & $-0,084$ & & $-0,233^{\star \star *}$ & $-0,197^{\star \star \star}$ \\
\hline & & 0,030 & 0,285 & & 0,060 & 0,074 \\
\hline \multirow[t]{2}{*}{ Industrialización (t-1) } & & & & & $-0,048$ & $-0,130$ ** \\
\hline & & & & & 0,061 & 0,052 \\
\hline \multirow[t]{2}{*}{ Industrialización (t-2) } & & & & & $0,119 * * \star$ & $0,118^{* \star *}$ \\
\hline & & & & & 0,032 & 0,027 \\
\hline \multirow[t]{2}{*}{ Cambio de gobierno } & $0,007^{*}$ & 0,007 & 0,009 * & & & 0,009 * \\
\hline & 0,004 & 0,018 & 0,005 & & & 0,005 \\
\hline \multirow[t]{2}{*}{ Crisis externa } & & & $-0,042^{* * *}$ & & & $-0,031^{\text {***}}$ \\
\hline & & & 0,007 & & & 0,012 \\
\hline
\end{tabular}

Fuente: Elaboración propia.

Nota: $\quad$ * significativo al $90 \%$; ${ }^{* \star}$ significativo al $95 \%$; ${ }^{* \star}$ significativo al $99 \%$. 